\title{
IImplementation of a Semi-Lagrangian Fully Implicit Time Integration of the Unified Soundproof System of Equations for Numerical Weather Prediction
}

\author{
Abdessamad Qaddouri, ${ }^{\mathrm{a}}$ Claude Girard, ${ }^{\mathrm{a}}$ Syed Zahid Husain, ${ }^{\mathrm{a}}$ And Rabah Aider ${ }^{\mathrm{a}}$ \\ a Atmospheric Numerical Prediction Research Section, Meteorological Research Division, Environment and Climate Change Canada, \\ Dorval, Quebec, Canada
}

(Manuscript received 3 September 2020, in final form 1 April 2021)

\begin{abstract}
An alternate dynamical core that employs the unified equations of A. Arakawa and C. S. Konor has been developed within Environment and Climate Change Canada's Global Environmental Multiscale (GEM) atmospheric model. As in the operational GEM dynamical core, the novel core utilizes the same fully implicit two-time-level semiLagrangian scheme for time discretization while the log-pressure-based terrain-following vertical coordinate has been slightly adapted. Overall, the new dynamical core implementation required only minor changes to the existing informatics code of the GEM model, and, from a computational performance perspective, the new core does not incur any significant additional cost. A broad range of tests - that include both two-dimensional idealized theoretical cases and threedimensional deterministic forecasts covering both hydrostatic and nonhydrostatic scales-have been carried out to evaluate the performance of the new dynamical core. For all of the tested cases, when compared with the operational GEM model, the new dynamical core based on the unified equations has been found to produce statistically equivalent results. These results imply that the unified equations can be adopted for operational numerical weather prediction that would employ a single soundproof system of equations to produce reliable forecasts for all meteorological scales of interest with negligible changes for the computational overhead.
\end{abstract}

KEYWORDS: Anelastic models; General circulation models; Nonhydrostatic models; Numerical weather prediction/forecasting; Semi-Lagrangian models

\section{Introduction}

The dynamical cores of the different atmospheric models are generally derived from the fully compressible Euler equations, often referred to as the elastic Euler (EE) system. Solutions of the EE equations contain modes of different spatiotemporal scales including the vertically propagating sound waves. Although the energy possessed by these sound waves is very low-and are therefore justifiably considered to be inconsequential for the atmospheric dynamics-they are numerically problematic. In particular, the vertically propagating sound waves impose severe time-step restrictions on the numerical weather prediction (NWP) models. Various approaches have therefore been pursued over the past many decades to derive approximate equations of atmospheric circulation that-for the scales of interest-filter out the vertically propagating sound waves associated with the EE system, while retaining the meteorologically important modes with negligible distortion. In general, these approximate equations are referred to as soundproof equations, although some of these formulations may retain the horizontally propagating Lamb waves.

At present, many operational atmospheric models-for both global and continental-scale regional predictions-mostly

\footnotetext{
Denotes content that is immediately available upon publication as open access.

Corresponding author: Abdessamad Qaddouri, abdessamad.qaddouri@ canada.ca
}

operate at resolutions that fall within the hydrostatic regime. The hydrostatic approximation, which filters out the acoustic waves by reducing the prognostic vertical momentum equation into a diagnostic hydrostatic relation, is found to be generally acceptable for these models. Such an approximation, however, works by assuming negligible acceleration in the vertical motion and therefore inevitably breaks down for the nonhydrostatic scales. Even with its limitations, the hydrostatic approximation has been successfully and widely used in operational NWP for global and regional systems (Ullrich et al. 2017).

Currently, a popular strategy for many operational NWP models is to employ two sets of equations for the hydrostatic and nonhydrostatic limits. Hydrostatic simulations are carried out by solving the traditional primitive equations based on the hydrostatic approximation, whereas for simulations where the nonhydrostatic effects are nonnegligible the fully compressible EE system is adopted. A possible option to avoid having this dual system of equations is to employ the EE system for predominantly hydrostatic simulations as well. Even though the semi-implicit approach slows down the fastmoving waves and the use of semi-Lagrangian advection can allow large time-steps, allowing the underlying EE system to generate acoustic waves can still prove to be restrictive for operational NWP models. Over the past decades, this has therefore led to a lot of research to come up with a more robust soundproof system that is capable of producing acceptable forecast for both hydrostatic and nonhydrostatic scenarios.

The sound waves at the nonhydrostatic scales can be filtered out from the EE system by making the simple Boussinesq 
approximation, which assumes density fluctuations to be negligible everywhere except for the buoyancy term in the vertical momentum equation. The resulting flow is incompressible and divergence-free where the original prognostic continuity equation,

$$
\frac{\partial \rho}{\partial t}+\nabla \cdot(\rho \mathbf{V})=0
$$

is replaced by a diagnostic relation given by

$$
\nabla \cdot \mathbf{V}=0
$$

where $\rho$ is the density, $\mathbf{V}$ is three-dimensional velocity vector, and $\nabla$ denotes the three-dimensional gradient operator. However, through scale analysis it can be shown that Eq. (2) is only acceptable as long as the vertical extent of circulation is lower than the density-scale height (Randall 2000). As a result, the Boussinesq approximation is more suitable for shallow oceanic circulations.

The anelastic approximation, originally coined by Ogura and Phillips (1962), may be considered as a further generalization of the Boussinesq approximation that extends the validity of the resulting soundproof equations to deeper atmospheric circulations. Although the equations resulting from the anelastic approximation are very similar to those proposed by Batchelor (1953), the systematic scale analysis presented by Ogura and Phillips (1962) identified two underlying limiting assumptions: (i) all deviations of potential temperature $\theta$ from a constant mean value of the actual potential temperature $\Theta$ are very small, and (ii) the only motions of interest are those with time scales similar to the gravity waves. As a result of these approximations, the continuity equation is reduced to a diagnostic relation that is slightly different from the Boussinesq approximation, and has the following form:

$$
\nabla \cdot(\bar{\rho} \mathbf{V})=0
$$

where $\bar{\rho}(z)$ is the vertically variable density associated with an adiabatically stratified reference state. Overall, the anelastic system conserves energy. Also, in the context of a linear hydrostatic mountain-wave problem, Nance and Durran (1994) demonstrated that the error attributable to the different components of an NWP model's numerics may be comparable to or larger than those due to the anelastic approximation. Similar conclusions were drawn by Smolarkiewicz et al. (2001) for global simulations. Nevertheless, larger deviations in potential temperature from the constant reference $\Theta$ make it unsuitable for phenomena like deep moist convection (Durran 1989). Furthermore, through normal mode analysis, Davies et al. (2003) have shown that the Rossby modes are significantly misrepresented by the different forms of anelastic approximation for scales that are of importance in NWP.

Durran (1989) later introduced the concept of a pseudoincompressible system where the compressible continuity equation within the EE system is replaced by the pseudoincompressible equation given by

$$
\nabla \cdot(\bar{\rho} \bar{\theta} \mathbf{V})=\frac{H}{c_{p} \bar{\pi}},
$$

where $\bar{\rho}(z)$ and $\bar{\theta}(z)$ are the density and potential temperature associated with a vertically varying mean state, $\bar{\pi}(z)$ is the corresponding Exner function, $H$ is the heating per unit volume, and $c_{p}$ is specific heat at constant pressure. Unlike the anelastic approximation, which completely neglects the perturbation density field, the pseudoincompressible approximation only neglects density perturbations related to the isentropic compressibility effect (Arakawa and Konor 2009) while retaining the effects of density perturbations arising due to the changes in temperature within the modified continuity equation. Relative to the anelastic approximation, the pseudoincompressible approach improves the prediction accuracy in the stratosphere while allowing energy conservation irrespective of the mean-state stratification. Although more work has been carried out for generalizing the pseudoincompressible approach (Durran 2008), the analyses by Davies et al. (2003) found the deeper vertical modes associated with large horizontal scales to be misrepresented by this approximation-making it only suitable for NWP applications at short horizontal scales.

A major breakthrough in the search of soundproof equations came through the work of Arakawa and Konor (2009) who proposed a unified approach to combine the concepts of hydrostatic approximation for the large scales and the anelastic approximation for the small scales. The resulting system, however, behaves similar to Durran's pseudocompressible system for the smallest scales (Arakawa and Konor 2009; Benacchio and Klein 2016). The underlying approximation is based on a reasonable assumption that any nonhydrostatic departure in the total density $\rho$ from its hydrostatic counterpart $\tilde{\rho}$ is negligible. This leads to a continuity equation of the following form:

$$
\frac{\partial \tilde{\rho}}{\partial t}+\nabla \cdot(\tilde{\rho} \mathbf{V})=0,
$$

which, similar to the anelastic and pseudocompressible systems, remains a diagnostic relation, as the hydrostatic density is not treated as a prognostic variable by this system. No other limiting approximation is made by this approach. By applying normal mode analysis Arakawa and Konor (2009) have shown that among all the soundproof systems, the unified approach leads to the best resemblance with the fully compressible EE system by improving the representation of the Rossby modes while preserving the fast Lamb modes. Konor (2014) later presented a dry dynamical core based on the unified system with a height-based vertical coordinate to demonstrate that this approach is capable of predicting dynamical processes involving a wide range of scales including cyclogenesis as well as convective-scale rising and sinking bubbles.

Recently, Voitus et al. (2019) adopted the unified approach of Arakawa and Konor (2009), and adapted the associated approximation to a mass-based terrain-following vertical coordinate. They chose to refer to the unified equations as a "quasi-elastic system," which appears to be a more reasonable 
terminology that is based on the nature of the pertinent approximation. Hereinafter, this approach will be referred to as the quasi-elastic (QE) approach throughout this article. Voitus et al. (2019) implemented the QE system within a semi-implicit spectral-transform model with an Eulerian transport scheme. The QE model was evaluated through a number of idealized test cases for simulating-among other scenarios-two-dimensional mountain waves and large-scale baroclinic instability. The results obtained with the QE model were found to be in very good agreement with the predictions from a fully compressible nonhydrostatic model. One of the limiting aspects of the QE model developed by Voitus et al. (2019) is the cost of the iterative enforcement of the QE divergence constraint, particularly due to the cost of inverse transforms required for checking its convergence. Although they have suggested an alternate approach based on redefining the prognostic variable in the vertical momentum equation to get around this issue, the stability of such a modification requires further analyses and testing.

The work presented in this article is primarily motivated by the QE model presented by Voitus et al. (2019). The objective of the present study is to first adopt the QE approach within Environment and Climate Change Canada's (ECCC) operational GEM model, which also uses a mass-based terrainfollowing coordinate (TFC) in the vertical direction. This coordinate, being based on log-hydrostatic pressure, is very amenable to the QE system by means of a slight modification in its definition. Furthermore, by implementing the QE approach within an operational NWP model, this article aims to demonstrate the effectiveness of the soundproof unified system of Arakawa and Konor (2009) for different idealized theoretical cases as well as for NWP systems at both the hydrostatic and nonhydrostatic scales. Another goal of this work is to demonstrate that the implementation of the QE approach in GEM does not lead to any significant additional computational cost for delivering predictions with similar accuracy with respect to the existing hydrostatic and nonhydrostatic versions of the GEM model.

This article is organized as follows. To begin with, a brief description of the GEM model is provided in section 2 followed by the presentation of the system of equations in GEM with respect to the operational log-hydrostatic-pressure-type TFC. In section 3 , the modifications to the vertical coordinate necessitated by the QE approach are presented along with the modified system of equations with respect to the new TFC and the QE approximation. Furthermore, section 4 demonstrates that the system of unified equations is free of vertically propagating sound waves. Following this, the numerical experiments and the associated results are presented in section 5, and section 6 summarizes the conclusions. The details of the derivation pertaining to the $\mathrm{QE}$ approach is provided in appendix $\mathrm{A}$, and the numerical solution procedure for the QE system of equations is presented in appendix B.

\section{Model description}

The Global Environmental Multiscale (GEM) model is used for operational forecasting at ECCC (Qaddouri and Lee 2011;
Girard et al. 2014; Husain and Girard 2017). At present, the GEM dynamical core employs two sets of equations for quasi-hydrostatic (GEM-QH) and nonhydrostatic (GEM-NH) dynamics.

\section{a. Governing equations}

The GEM model governing equations of the nonhydrostatic atmosphere, which is based on the fully compressible EE system, when transformed to a generalized vertical $\zeta$ coordinate, can be written as follows:

$$
\begin{gathered}
\frac{d \mathbf{V}_{h}}{d t}+f \mathbf{k} \times \mathbf{V}_{h}+R T\left[\nabla_{\zeta} \ln p-\left(\nabla_{\zeta} z\right)\left(\frac{\partial z}{\partial \zeta}\right)^{-1} \frac{\partial \ln p}{\partial \zeta}\right]=\mathbf{F}_{h} \\
\frac{d w}{d t}+R T\left(\frac{\partial z}{\partial \zeta}\right)^{-1} \frac{\partial \ln p}{\partial \zeta}+g=F_{w} \\
\frac{d}{d t} \ln \left(\rho \frac{\partial z}{\partial \zeta}\right)+\nabla_{\zeta} \cdot \mathbf{V}_{h}+\frac{\partial \dot{\zeta}}{\partial \zeta}=F_{\rho} \\
\frac{d \ln T}{d t}-\kappa \frac{d \ln p}{d t}=F_{T} \\
\frac{d z}{d t}-w=0 \\
\rho=\frac{p}{R T}, \quad \text { and } \\
z=z\left(\zeta, \mathbf{r}_{h}, t\right)
\end{gathered}
$$

where $\left(\mathbf{V}_{h}, w\right)$ are the horizontal and vertical velocity components, $\rho$ is the air density, $T$ is the temperature, $p$ is the pressure, $\dot{\zeta}$ is the generalized vertical velocity, and $z$ is the geometric height; $\nabla_{\zeta}$ denotes the horizontal gradient operator along $\zeta$ and $d / d t$ denotes the material derivative. Also in the above equations, $f$ represents the Coriolis parameter, $R$ is the gas constant, and $\kappa=R / c_{p}$, with $c_{p}$ being the specific heat at constant pressure. Equations (6)-(9) are referred to as the horizontal momentum, vertical velocity, continuity and thermodynamic equations, respectively. Equation (10) describes the relation between the two vertical velocities, whereas Eqs. (11) and (12) are the equation of state and the diagnostic equation defining $\zeta$. Overall, we have eight equations with eight dependent variables $\left(\mathbf{V}_{h}, w, T, \rho, p, \dot{\zeta}, z\right)$ and four independent variables $\left(t, \mathbf{r}_{h}, \zeta\right)$. The terms $\mathbf{F}_{h}, F_{w}, F_{\rho}$, and $F_{T}$ on the right-hand side (rhs) represent the subgrid-scale physical forcings whose effects are parameterized. For the remaining discussion, all of the physical forcing terms will be made to vanish in what constitutes a formulation of the inviscid and adiabatic GEM dynamical core.

\section{b. Vertical coordinate}

The description of the vertical coordinate presented in this section largely follows that of Husain et al. (2019). The loghydrostatic-pressure terrain-following vertical coordinate of the operational GEM model (Girard et al. 2014; Husain et al. 2020) takes the form

$$
\ln \bar{p}=\zeta+B s,
$$


where $\zeta=\ln \left(\eta p_{\text {ref }}\right)$ now represents the terrain-following vertical coordinate with $\eta$ being a user-defined nondimensional variable such that $p_{\text {top }} / p_{\text {ref }}<\eta \leq 1$, and $p_{\text {ref }}=10^{5} \mathrm{~Pa}$ is a reference pressure. Furthermore, $\bar{p}$ is the hydrostatic pressure, where $\partial \bar{p} / \partial z=-g \rho ; B$ depends only on $\zeta$ to determine the rate of vertical decay of terrain imprints, with $B\left(\zeta_{s}\right)=$ $1 \geq B(\zeta) \geq B\left(\zeta_{T}\right)=0$; and $s=\ln \left(\bar{p}_{s} / p_{\text {ref }}\right)$ is the mass variable that depends on the surface pressure $\bar{p}_{s}$. At the surface, $\eta=1$ and therefore $\zeta_{s}=\ln p_{\text {ref. }}$ Equation (13) can also therefore be expressed as

$$
\ln \left(\bar{p} / p_{\text {ref }}\right)=\ln \eta+B s .
$$

The above $\zeta$ coordinate follows the idea of the generalized hydrostatic-pressure hybrid coordinate presented by Laprise (1992), and additional details concerning its definition are provided in the existing literature (Girard et al. 2014; Husain et al. 2020).

\section{c. GEM formulation}

The formulation of the GEM equations in the terrainfollowing $\zeta$ coordinate starts by defining an isothermal and stationary basic-state that is at a hydrostatic balance. Therefore, for a basic-state temperature $T_{*}=240 \mathrm{~K}$, the corresponding geopotential profile $\phi_{*}$ is determined by

$$
\frac{\partial \phi_{*}}{\partial \zeta}=-R T_{*} .
$$

Later, the air density is eliminated as a prognostic variable through the application of the hydrostatic relation $\partial \bar{p} / \partial z=-g \rho$. A transformation of the emanating equations from the generalized vertical coordinate to the terrain-following $\zeta$-coordinate, as in Girard et al. (2014), then changes the system of Eqs. (6)(12) as follows:

$$
\begin{aligned}
& \frac{d \mathbf{V}_{h}}{d t}+f \mathbf{k} \times \mathbf{V}_{h}+R T \nabla_{\zeta}(B s+q)+(1+\mu) \nabla_{\zeta} \phi^{\prime}=0, \\
& \frac{d w}{d t}-g \mu=0 \\
& \frac{d}{d t}\left[B s+\ln \left(1+\frac{\partial B}{\partial \zeta} s\right)\right]+\nabla_{\zeta} \cdot \mathbf{V}_{h}+\frac{\partial \dot{\zeta}}{\partial \zeta}+\dot{\zeta}=0, \\
& \frac{d}{d t} \ln \left(\frac{T}{T_{*}}\right)-\kappa\left[\frac{d}{d t}(B s+q)+\dot{\zeta}\right]=0, \\
& \frac{d \phi^{\prime}}{d t}-R T_{*} \dot{\zeta}-g w=0, \\
& \frac{T}{T_{*}}-e^{q}\left\{1-\frac{\frac{\partial\left[\phi^{\prime}+R T_{*}(B s)\right]}{\partial \zeta}}{R T_{*}\left(1+\frac{\partial B}{\partial \zeta} s\right)}\right\}=0, \\
& 1+\mu-e^{q}\left(1+\frac{\frac{\partial q}{\partial \zeta}}{1+\frac{\partial B}{\partial \zeta} s}\right)=0
\end{aligned}
$$

where $q=\ln (p / \bar{p})$ is the nonhydrostatic pressure perturbation, $\mu=[(p / \bar{p})(\partial \ln p / \partial \ln \bar{p})]-1$ is the ratio of the vertical acceleration to the gravitational acceleration, and $\phi^{\prime}=\phi-$ $\phi_{*}$ is the geopotential perturbation with respect to the reference state geopotential $\phi_{*}(\zeta)=-R T_{*}\left(\zeta-\zeta_{s}\right)$, which is computed by integrating Eq. (15) with the boundary condition $\phi_{*}\left(\zeta_{s}\right)=0$.

The complete system of equations involves a total of eight equations-six prognostic Eqs. (16)-(20) and two diagnostic Eqs. (21) and (22) - for eight unknown variables $u, v, w, T, q$, $(\dot{\zeta}, s), \phi^{\prime}, \mu$. Since $s$ is a two-dimensional variable related only to the surface pressure and the three-dimensional variable $\dot{\zeta}$ vanishes at the surface, the combination $(\dot{\zeta}, s)$ is considered as a three-dimensional variable of the model.

\section{The QE formulation of GEM with a modified TFC}

Before introducing the QE approximation in GEM, we need to define a new mass-based terrain-following vertical coordinate by slightly modifying the definition of the $\zeta$ coordinate of the existing GEM model. Instead of using $\partial \bar{p} / \partial z=-g \rho$, where $\bar{p}$ represents the hydrostatic pressure and $\rho$ is the true density, we now use

$$
\frac{\partial \tilde{\pi}}{\partial z}=-\frac{g}{c_{p} \theta},
$$

where $\tilde{\pi}=\left(\tilde{p} / p_{o}\right)^{\kappa}$ is the Exner function of a hydrostatic state and $\theta=T\left(p_{o} / p\right)^{\kappa}$ is the true potential temperature. The crucial point here is the assumption that $\theta=\tilde{\theta}$, where $\tilde{\theta}=\tilde{T}\left(p_{o} / \tilde{p}\right)^{\kappa}$. In other words, $\theta=\tilde{\theta}$ is the only link between the true state represented by $p=\rho R T$ and the newly defined quasi-hydrostatic state with $\tilde{p}=\tilde{\rho} R \tilde{T}$. This leads to

$$
\tilde{\rho}=\frac{\tilde{p}}{R T}\left(\frac{p}{\tilde{p}}\right)^{\kappa},
$$

and Eq. (23) gives

$$
\frac{\partial \tilde{p}}{\partial z}=-g \tilde{\rho}
$$

As noted by Voitus et al. (2019), these two equations indicate that $\tilde{p}$ and $\tilde{\rho}$ are dependent on the potential temperature of the true state and are in hydrostatic balance. Now, by redefining the variable $q=\ln (p / \tilde{p})$ as representing the departure from the quasi-hydrostatic pressure $\tilde{p}$, we can relate the true state $(T, p, \rho)$ to the quasi-hydrostatic state $(\tilde{T}, \tilde{p}, \tilde{\rho})$ as

$$
\begin{aligned}
& p=\tilde{p} e^{q}, \\
& T=\tilde{T} e^{\kappa q}, \quad \text { and } \\
& \rho=\tilde{\rho} e^{(1-\kappa) q} .
\end{aligned}
$$

The quasi-hydrostatic pressure $\tilde{p}$ can then be used to define a new mass-based terrain-following vertical coordinate as

$$
\ln \tilde{p}=\zeta+B s,
$$


where we have kept the same $\zeta$ notation to define the new vertical coordinate, as in the current GEM equations. The metric term $B$ remains unchanged, and $s=\ln \left(\tilde{p}_{s} / p_{\text {ref }}\right)$ is the modified mass variable, which is now related to the value of $\tilde{p}$ at the surface.

The nonhydrostatic GEM equations in this new TFC (see appendix A for further details) may be formulated as follows:

$$
\begin{aligned}
& \frac{d \mathbf{V}_{h}}{d t}+f \mathbf{k} \times \mathbf{V}_{h}+R T \nabla_{\zeta}(B s+q)+(1+\mu) \nabla \phi^{\prime}=0, \\
& \frac{d w}{d t}-g \mu=0, \\
& \frac{d}{d t}\left[B s+\ln \left(1+\frac{\partial B}{\partial \zeta} s\right)\right]+\nabla_{\zeta} \cdot \mathbf{V}_{h}+\frac{\partial \dot{\zeta}}{\partial \zeta}+\dot{\zeta}+(1-\kappa) \frac{d q}{d t}=0 \\
& \frac{d}{d t} \ln \left(\frac{T}{T_{*}}\right)-\kappa\left[\frac{d}{d t}(B s+q)+\dot{\zeta}\right]=0, \\
& \frac{d \phi^{\prime}}{d t}-R T_{*} \dot{\zeta}-g w=0, \\
& \frac{T}{T_{*}}-e^{\kappa q}\left\{1-\frac{\frac{\partial\left[\phi^{\prime}+R T_{*}(B s)\right]}{\partial \zeta}}{R T_{*}\left(1+\frac{\partial B}{\partial \zeta} s\right)}\right\}=0, \quad \text { and } \\
& 1+\mu-e^{\kappa q}\left(1+\frac{\frac{\partial q}{\partial \zeta}}{1+\frac{\partial B}{\partial \zeta} s}\right)=0
\end{aligned}
$$

Although the total number of equations and unknowns remain the same, the variables of the model are now defined with respect to the new $\zeta$ coordinate. The only difference between this formulation of the GEM equations and the existing formulation (in the previous section) reside in three equations: the two diagnostic Eqs. (35) and (36) (hydrostatic equation and $\mu$ equation) where $q$ is replaced by $\kappa q$ in the exponential, and the continuity Eq. (32), which includes an additional term $(1-\kappa) d q / d t$ involving the nonhydrostatic pressure tendency.

No approximation has yet been made. The quasi-elastic approximation now simply requires the last term $(1-\kappa) d q / d t$ in the continuity Eq. (32) to be neglected. Consequently, the mass continuity equation in the $\mathrm{QE}$ system of equations becomes

$$
\frac{d}{d t}\left[B s+\ln \left(1+\frac{\partial B}{\partial \zeta} s\right)\right]+\nabla_{\zeta} \cdot \mathbf{V}_{h}+\frac{\partial \dot{\zeta}}{\partial \zeta}+\dot{\zeta}=0
$$

and, as noted by Durran (2008) and Arakawa and Konor (2009), the suppression of the feedback between the nonhydrostatic log-pressure perturbation $q$ and the velocity divergence, gives rise to a soundproof nonhydrostatic system of equations. Section 4 shows that this GEM-QE system of equations [Eqs. (30), (31), (33)-(36), and (37)] does not contain the vertically propagating sound waves. The numerical solution method for this new dynamical core based on the QE equations is similar to the one used in the current operational GEM model. The relevant details are presented in appendix B.

The choice of the vertical coordinate and the thermodynamic variables in GEM-QE not only resulted in maintaining the mass-continuity equation in the same form as in the fully compressible GEM model but also the rest of the prognostic equations. This permits us to keep the temporal integration procedure for GEM-QE similar to that already in place in operational model.

\section{Absence of sound waves in the linear QE system}

It is possible to show that the linear system of equations resulting from the QE approximation is free of vertically propagating sound waves. The linear system of equations can be written as

$$
\begin{aligned}
\frac{\partial \mathbf{V}_{h}}{\partial t}+\nabla_{\zeta}\left[\phi^{\prime}+R T_{*}(B s+q)\right] & =0, \\
\frac{\partial w}{\partial t}-g \mu & =0, \\
\frac{\partial}{\partial t}\left[\left(B+\frac{\partial B}{\partial \zeta}\right) s\right]+\nabla_{\zeta} \cdot \mathbf{V}_{h}+\frac{\partial \dot{\zeta}}{\partial \zeta}+\dot{\zeta} & =0, \\
\frac{\partial}{\partial t}\left[\frac{T^{\prime}}{T_{*}}-\kappa(B s+q)\right]-\kappa \dot{\zeta} & =0, \\
\frac{\partial \phi^{\prime}}{\partial t}-R T_{*} \dot{\zeta}-g w & =0, \\
\frac{1}{T_{*}}+\frac{\partial \phi^{\prime}}{R T_{*}}-\kappa q+\frac{\partial B}{\partial \zeta} s & =0, \quad \text { and } \\
\mu-\left(\frac{\partial q}{\partial_{\zeta}}+\kappa q\right) & =0 .
\end{aligned}
$$

Introducing $P=\phi^{\prime}+R T_{*}(B s+q)$ and $Y=\dot{\zeta}+B(\partial s / \partial t)$ and eliminating $\mathbf{V}_{h}, \mu$, and $T^{\prime}$, we first reduce the system to four equations with four variables $w, q, P$, and $Y$ :

$$
\begin{aligned}
\frac{\partial w}{\partial t}-g\left(\frac{\partial}{\partial \zeta}+\kappa\right) q & =0, \\
-\frac{1}{R T_{*}} \frac{\partial}{\partial t} \frac{\partial P}{\partial \zeta}+\frac{\partial}{\partial t} \frac{\partial q}{\partial \zeta}-\kappa Y & =0, \\
-\nabla_{\zeta}^{2} P+\frac{\partial}{\partial t}\left(\frac{\partial Y}{\partial \zeta}+Y\right) & =0, \quad \text { and } \\
\frac{1}{R T_{*}} \frac{\partial P}{\partial t}-Y-\frac{\partial q}{\partial t}-\frac{g}{R T_{*}} w & =0 .
\end{aligned}
$$

Eliminating $q$ and $w$, we get

$$
-\left(\frac{\partial^{2}}{\partial t^{2}}+N_{*}^{2}\right)\left(\frac{\partial}{\partial \zeta}+\kappa\right) Y-\left(\frac{g}{R T_{*}}\right)^{2} \frac{\partial}{\partial \zeta}\left(\frac{\partial}{\partial \zeta}+\kappa\right) \frac{\partial P}{\partial t}=0 \quad \text { and }
$$




\section{a)}

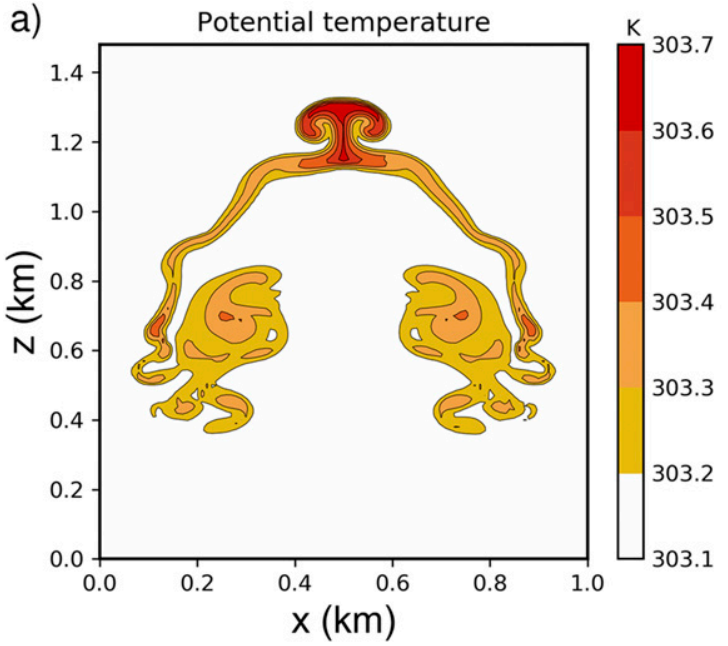

b)

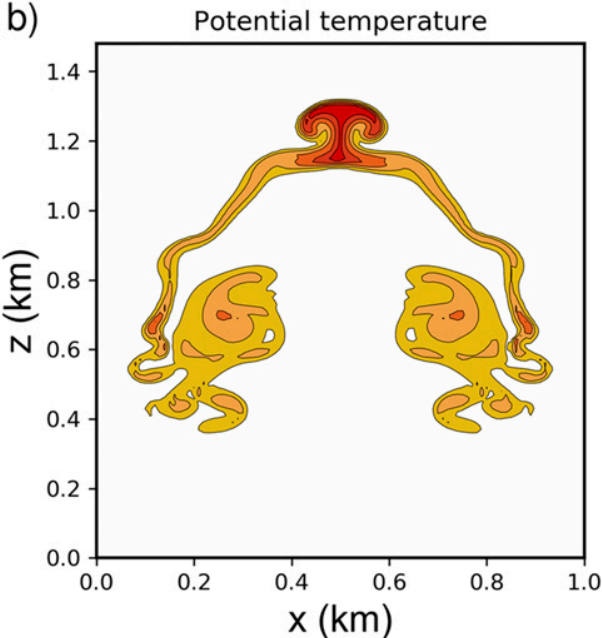

FIG. 1. Gaussian bubble after $18 \mathrm{~min}$ as predicted by (a) GEM-NH and (b) GEM-QE.

$$
-\nabla_{\zeta}^{2} P+\left(\frac{\partial}{\partial \zeta}+1\right) \frac{\partial Y}{\partial t}=0
$$

where $N_{*}^{2}=g^{2} /\left(R T_{*}\right)$. By eliminating $Y$, we get

$$
\left(\frac{\partial^{2}}{\partial t^{2}}+N_{*}^{2}\right) \nabla_{\zeta}^{2} P+\frac{1}{H_{*}^{2}}\left(\frac{\partial}{\partial \zeta}+1\right) \frac{\partial^{2}}{\partial t^{2}} \frac{\partial P}{\partial \zeta}=0 .
$$

Considering a solution of the form $\exp [i(k x+l y+n z-\omega t)]$, Eq. (51) is reduced to

$$
\left(-\omega^{2}+N_{*}^{2}\right)\left(-k^{2}-l^{2}\right)+\frac{1}{H_{*}^{2}}(i n+1) i n \omega^{2}=0
$$

where $H_{*}=R T_{*} / g$ is the thermodynamic height scale. By setting in $=i m H_{*}-(1 / 2)$, we obtain

$$
\left(-\omega^{2}+N_{*}^{2}\right)\left(-k^{2}-l^{2}\right)-\left(-m^{2}-1 / 4 H_{*}^{2}\right) \omega^{2}=0,
$$

which is the frequency equation for an incompressible medium. This equation is only of second order and only contains the gravity waves, whose frequency is given by

$$
\omega= \pm \sqrt{\left\{\frac{N_{*}^{2}\left(k^{2}+l^{2}\right)}{\left[k^{2}+l^{2}+m^{2}+1 /\left(4 H_{*}^{2}\right)\right]}\right\}} .
$$

\section{Numerical experiments}

The performance of the new QE dynamical core with the semi-Lagrangian fully implicit method is evaluated through two-dimensional idealized test cases involving a rising bubble of warm air (Robert 1993), a sinking bubble of cold air (Straka et al. 1993) and nonhydrostatic mountain waves (Schär et al. 2002) along with three-dimensional global and regional NWP systems. During these evaluations the predictions by the current GEM dynamical core are used as references for comparison.

\section{a. Evolution of a warm Gaussian bubble}

One of the two-dimensional test cases proposed by Robert (1993) is used here to test the ability of the new QE dynamical core to represent the advection and buoyancy effects in the
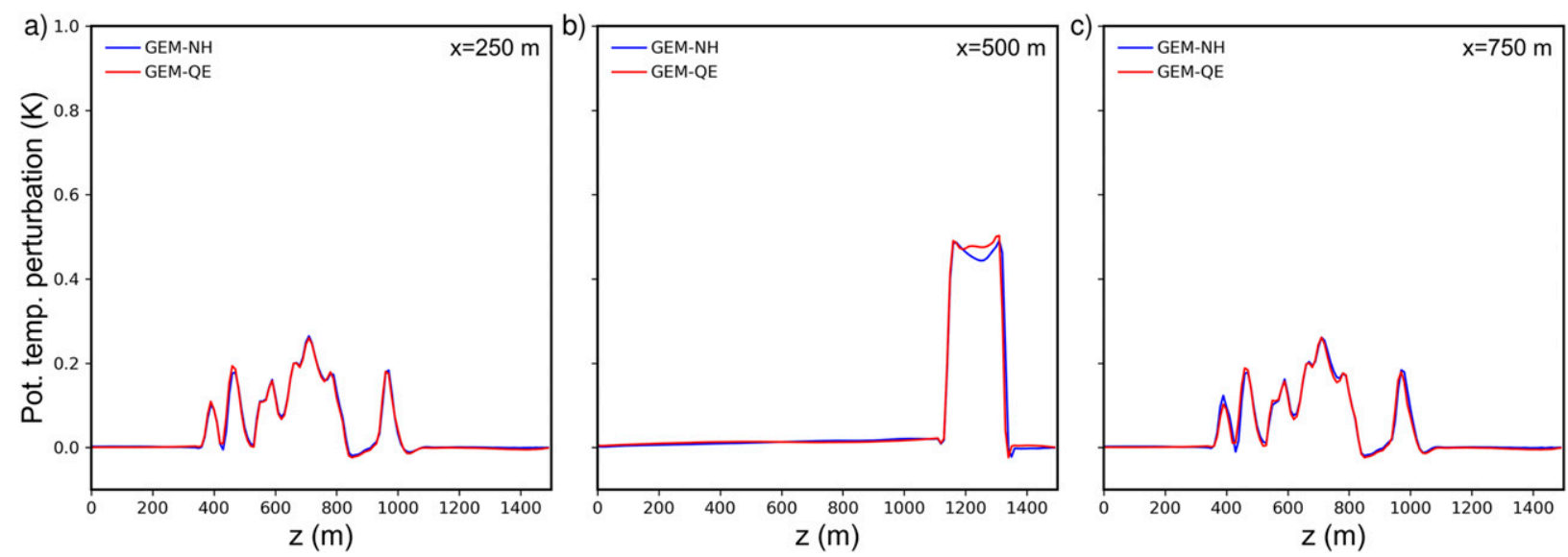

FIG. 2. Vertical profiles of potential temperature perturbation for the warm bubble at $t=18 \mathrm{~min}$ at $x=$ (a) 250 , (b) 500 , and (c) $750 \mathrm{~m}$. 

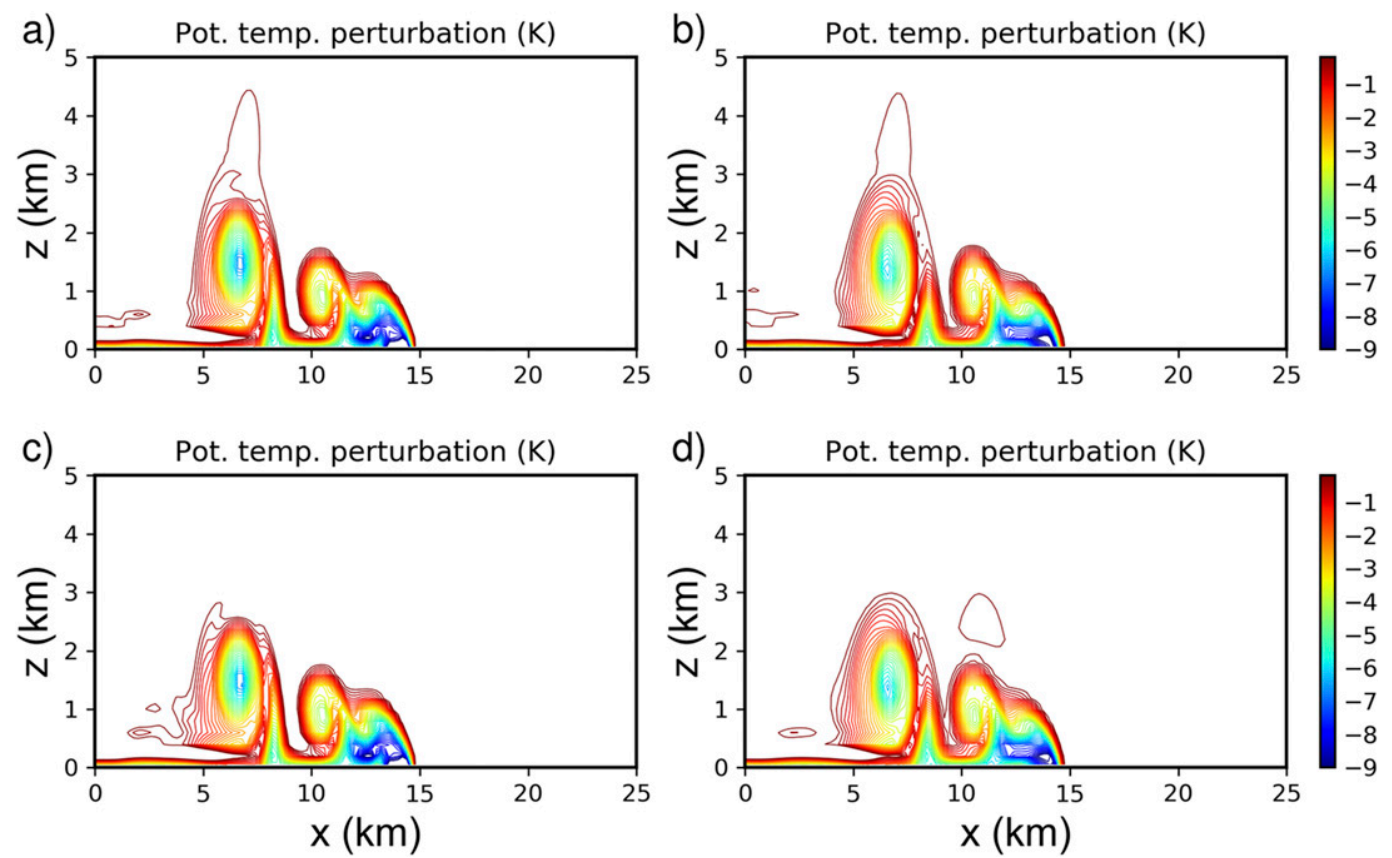

FIG. 3. Density current at $t=900 \mathrm{~s}$ as predicted by: (a),(b) GEM-NH and (c),(d) GEM-QE. In (a) and (c) no explicit horizontal diffusion is applied, whereas in (b) and (d) a horizontal Laplacian diffusion operator with a diffusion coefficient $K=100 \mathrm{~m}^{2} \mathrm{~s}^{-1}$ is applied to the $u, w$, and $\theta$ variables.

absence of any orography. In this test a warm bubble with an initial Gaussian distribution of potential temperature, within a dry isentropic atmosphere $(303.15 \mathrm{~K})$ is rising subjected to a small buoyancy force. The bubble is initially placed at $z_{o}=$ $260 \mathrm{~m}$ inside a closed box of 1000-m width and 1500-m height. It is important to note that no off-centering is applied in this experiment, and a time step of $6 \mathrm{~s}$ with a spatial resolution of $10 \mathrm{~m}$ is used. The state of the bubble after $18 \mathrm{~min}$ is presented in
Fig. 1 for both dynamical cores. The results predicted by the two dynamical cores are qualitatively equivalent in terms of shape, as well as symmetry. This closeness between the two predictions at an advanced time during the evolution of the bubble, implies that the use of the QE approximation instead of the current GEM nonhydrostatic dynamical core, has no negative impact on flow situations that are primarily driven by buoyancy and advection.
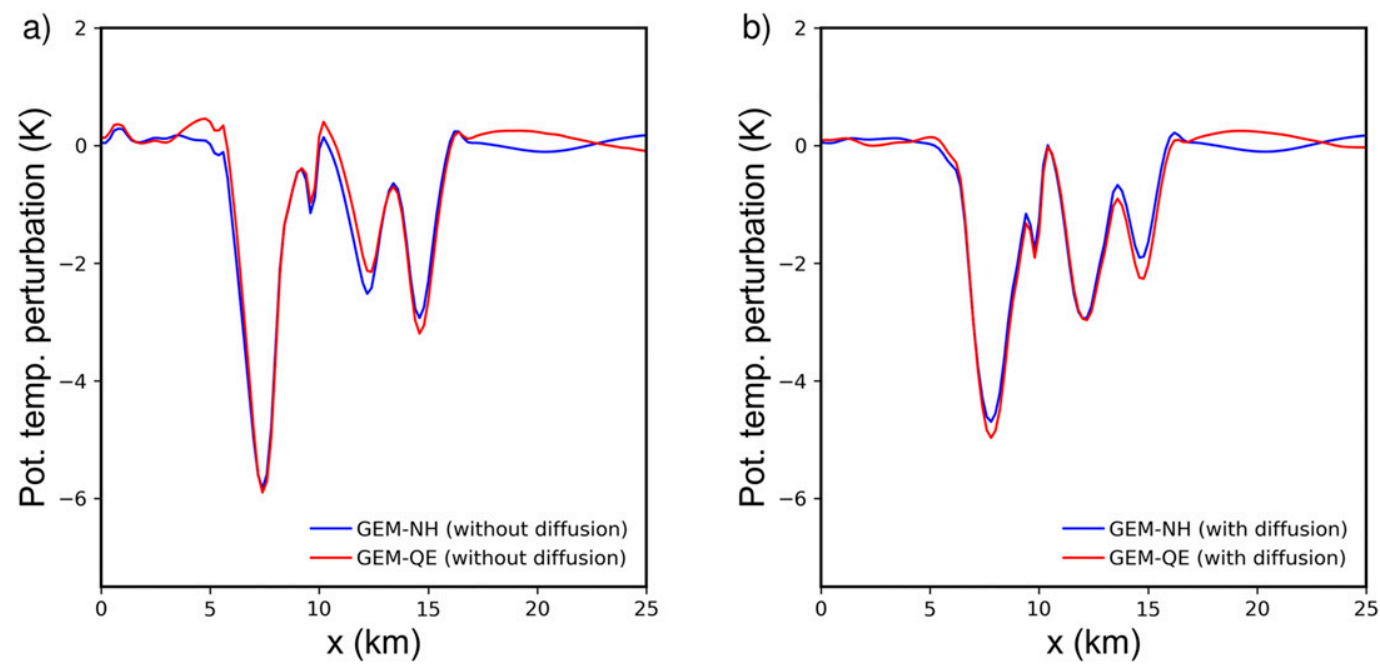

FIG. 4. The horizontal profiles of potential temperature perturbation at $1200-\mathrm{m}$ height for the cold bubble at $t=900 \mathrm{~s}$ for both GEM-NH and GEM-QE (a) without diffusion and (b) with diffusion. 
a)

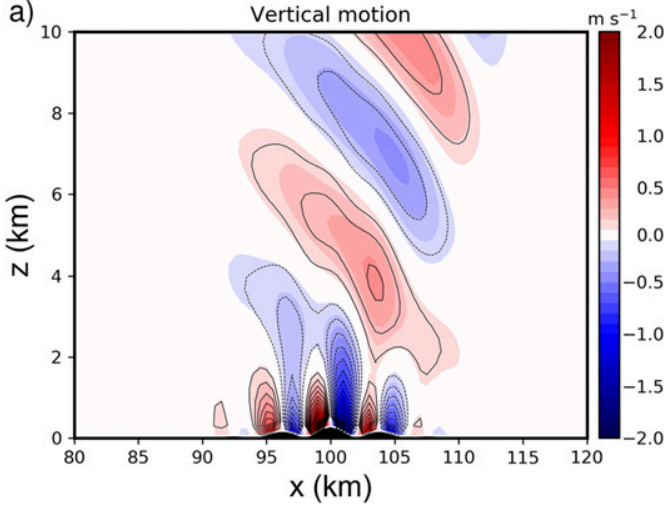

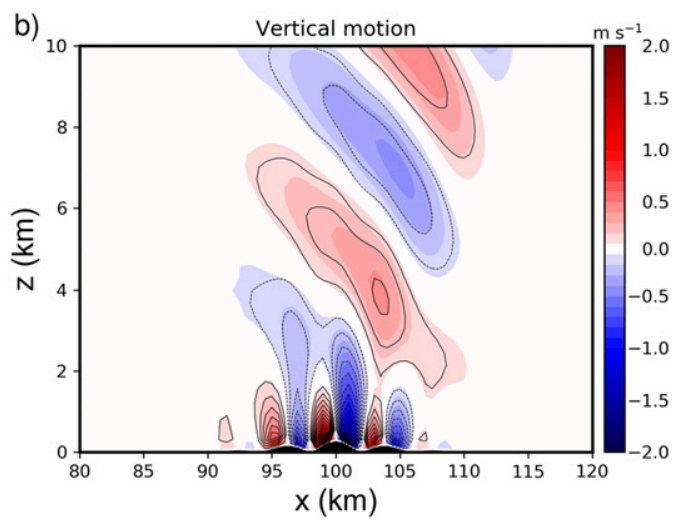

FIG. 5. Vertical velocity filled contours (with $0.1 \mathrm{~m} \mathrm{~s}^{-1}$ intervals) for the nonhydrostatic mountain waves case as predicted by (a) GEM-NH and (b) GEM-QE. The analytical solution is represented by solid (positive values) and dashed (negative values) contours.

To have a more quantitative comparison between the two dynamical cores, Fig. 2 presents the one-dimensional profiles of potential temperature along the vertical direction at $x=250,500$, and $750 \mathrm{~m}$. Apart from the small differences seen in the potential temperature at $x=500 \mathrm{~m}$ (along the stem of the mushroom developed at the top of the deformed bubble), the 3 profiles for GEM-NH and GEM-QE are very similar. The symmetry of the temperature profiles at $x=250$ and $750 \mathrm{~m}$ is also in excellent agreement. Overall, these results demonstrate
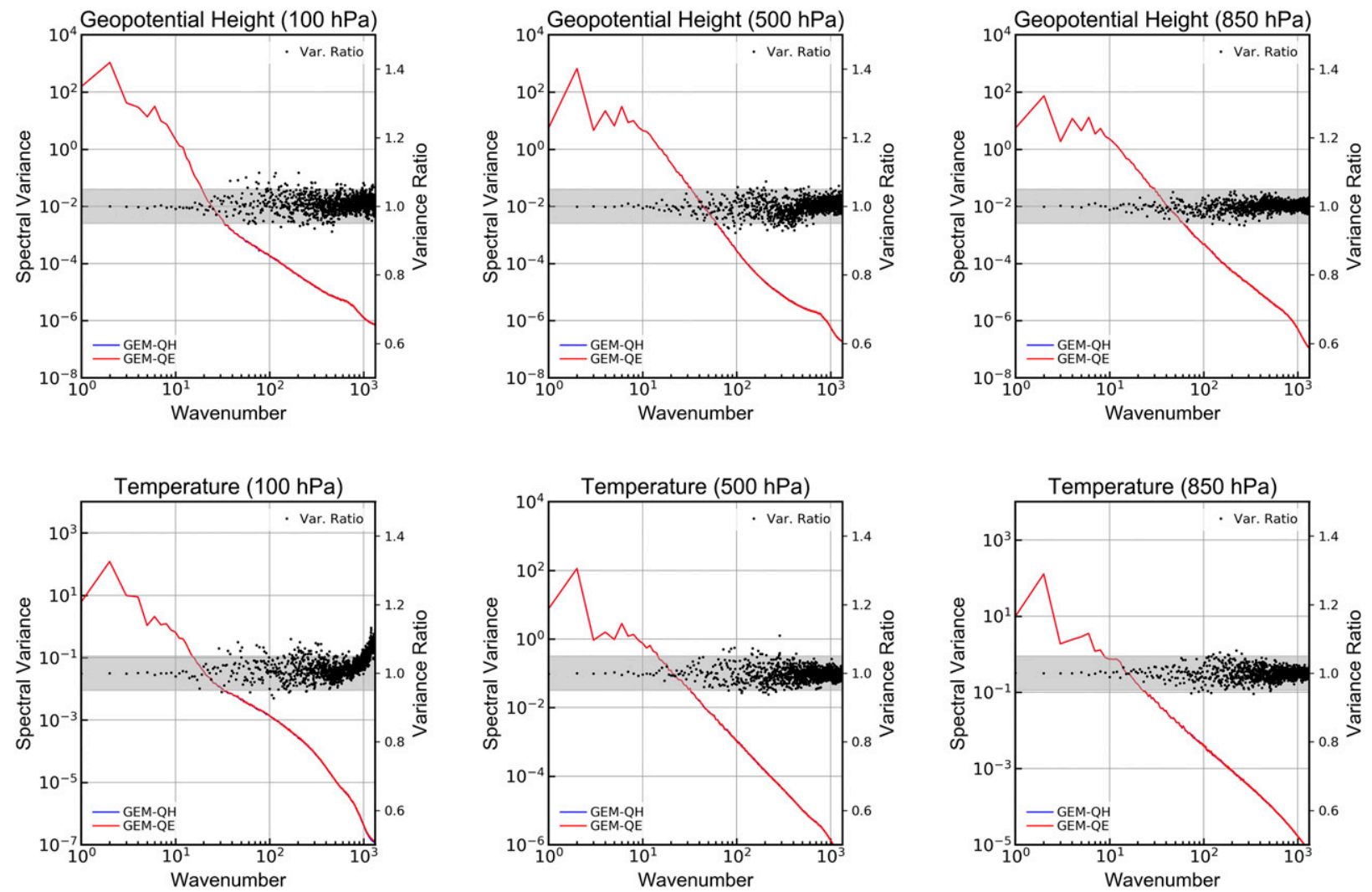

FIG. 6. Spectral variance of (top) geopotential height GZ and (bottom) temperature TT for a forecast lead time of $120 \mathrm{~h}$ with the GDPS (obtained by averaging the spectra for 10 winter forecasts) at three different pressure levels. The black circles in the figure denote variance ratios between GEM-QE and GEM-QH for different wavenumbers. The shaded area in the figure represents the region bounded by variance ratios of 0.95 and 1.05 , and, consequently, for most length scales the change of variance with GEM-QE is approximately within $5 \%$ of the reference GEM-QH. 

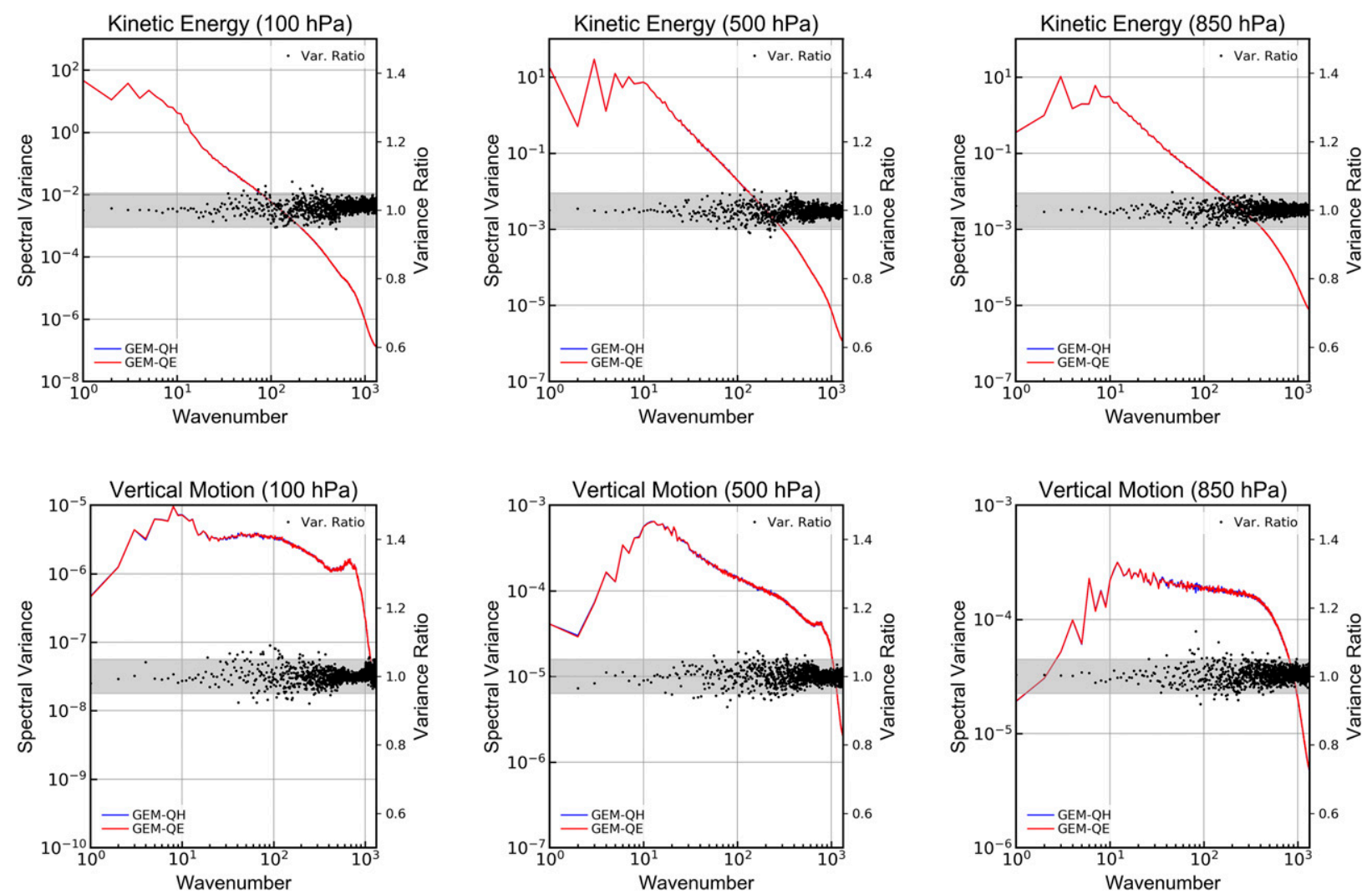

FIG. 7. Spectral variance of (top) kinetic energy KE and (bottom) vertical velocity WW for a forecast lead time of $120 \mathrm{~h}$ with GDPS. All other conditions are as in Fig. 6.

that for buoyancy driven flows GEM-QE is equivalent of GEM-NH.

\section{b. Evolution of a cold sinking bubble}

This is another idealized test case that has been used in the past to evaluate dynamical cores (Giraldo and Restelli 2008). In this test, an initial cold bubble of air is dropped in a neutrally stratified atmosphere leading to a sinking bubble that hits the lower boundary at a subsequent time. Interactions of the sinking bubble with the model surface make this case particularly challenging. Once the bubble hits the surface it starts to spread out laterally and forms three Kelvin-Helmholtz shear instability rotors over time. The potential temperature perturbation is defined as

$$
\theta^{\prime}=\frac{\theta_{c}}{2}[\cos (\pi r)+1]
$$

where $\theta_{c}=-15^{\circ} \mathrm{C}$ and $r$ is given by

$$
r=\sqrt{\left(\frac{x-x_{c}}{x_{r}}\right)^{2}+\left(\frac{z-z_{c}}{z_{r}}\right)^{2}},
$$

with the size of the bubble being defined by the parameters $\left(x_{r}\right.$, $\left.z_{r}\right) \equiv(4 \mathrm{~km}, 2 \mathrm{~km})$. The center of the bubble is initially placed at $\left(x_{c}, z_{c}\right) \equiv(0 \mathrm{~km}, 3 \mathrm{~km})$ inside a computational domain that is $51.2 \mathrm{~km}$ wide and $6.4 \mathrm{~km}$ high. Again, no off-centering is applied in this experiment, and a time step of $5 \mathrm{~s}$ with a spatial resolution of $200 \mathrm{~m}$ is used.

Figure 3 presents the potential temperature perturbation contours for this case after $900 \mathrm{~s}$ for both GEM-NH and GEM-QE. This figure shows the results without any diffusion and also with numerical diffusion applied to the $u, w$, and $\theta$ variables (with a diffusion coefficient $K=100 \mathrm{~m}^{2} \mathrm{~s}^{-1}$ ). In this figure, the 3 rotors are clearly visible and exhibit qualitatively similar characteristics for GEM-NH and GEM-QE models.

In addition, Fig. 4 shows the one-dimensional profile of the potential temperature perturbation along the horizontal direction at a height of $1200 \mathrm{~m}$ for both models. The results obtained by the two models show three negative wells in the profile of the potential temperature perturbation that represent the three distinct rotors. As can be seen, the temperature gradients as well as the minimum and maximum temperatures across the rotors for the two models are in very good agreements. Moreover, these results are comparable to the previously published results for other models (Giraldo and Restelli 2008).

\section{c. Nonhydrostatic mountain waves}

This two-dimensional test-case-presented by Schär et al. (2002), for which a quasi-linear analytical solution is available-is used here to examine the nonhydrostatic responses of the new dynamical core based on the QE approximation, in the 

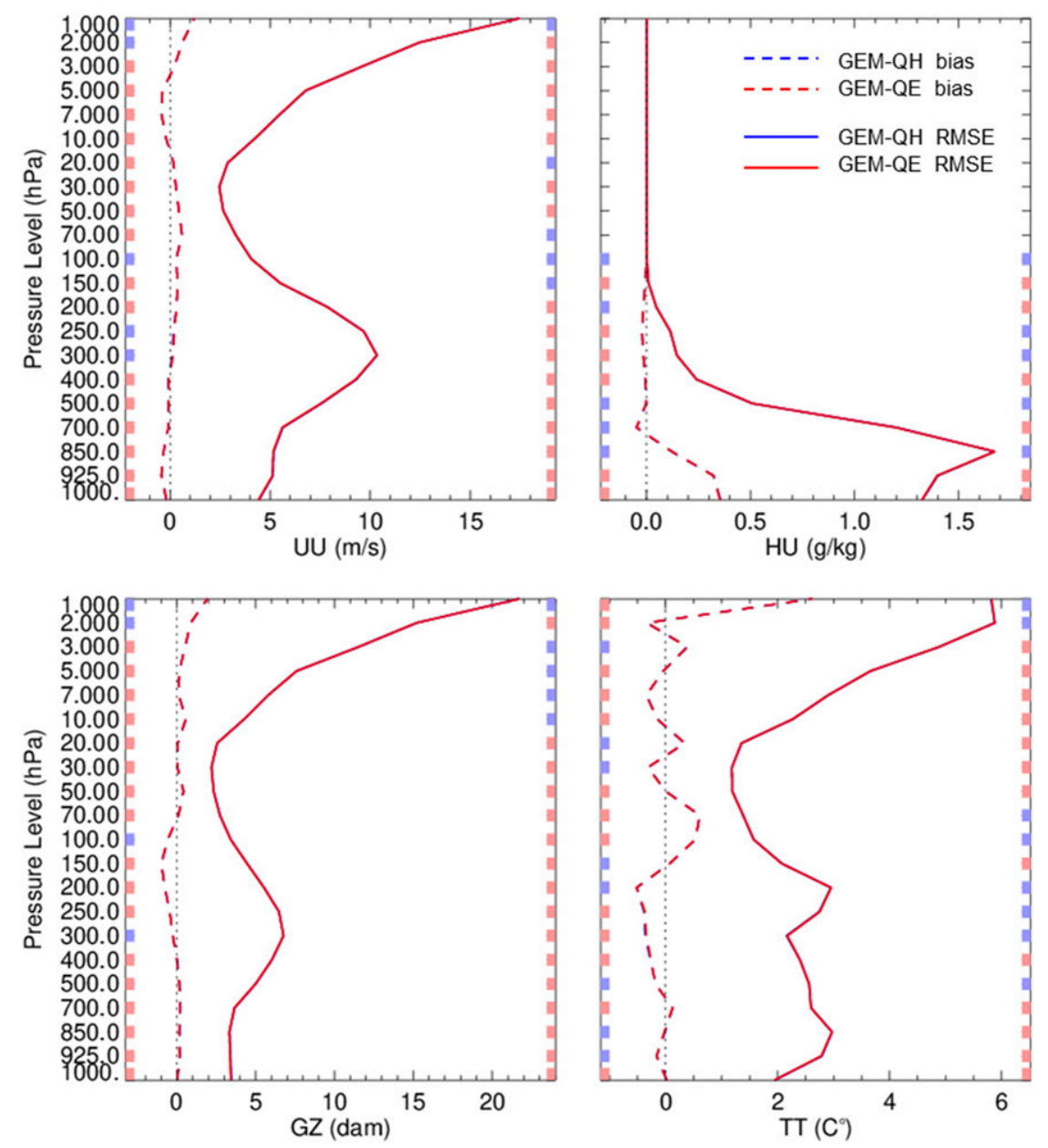

FIG. 8. Comparison of 120-h forecasts with GDPS with ERA5 analysis with GEM-QH (blue lines) and GEM-QE (red lines) for (top left) zonal wind, (top right) humidity, (bottom left) geopotential height, and (bottom right) temperature. The RMSE (solid lines) and bias (dashed lines) are shown. Verification is performed for a set of 44 integrations over the Northern Hemisphere winter period: 16 Dec 2016-18 Feb 2017.

presence of idealized mountains. This test simulates the generation of gravity waves by a constant horizontal flow over a mountain range within a uniform stratified atmosphere.

The orography is defined as $h(x)=h_{o} \exp \left[-(x / a)^{2}\right] \cos ^{2}(\pi x / b)$, where $h_{o}=250 \mathrm{~m}, a=5 \mathrm{~km}$, and $b=4 \mathrm{~km}$. The upstream flow is defined by horizontal velocity $U=10 \mathrm{~m} \mathrm{~s}^{-1}$, a surface temperature of $288 \mathrm{~K}$, a surface pressure $p_{o}=1000 \mathrm{hPa}$, and a constant buoyancy frequency $N=0.01 \mathrm{~s}^{-1}$. A time step of $32 \mathrm{~s}$ is used, and no off-centering is applied. All other conditions for this test case are similar to those presented by Husain and Girard (2017). The analytical solution of the linearized problem is composed of large-scale hydrostatic waves with deep vertical propagation and small-scale nonhydrostatic waves that decay rapidly while remaining close to the surface. As shown in Fig. 5, the solutions produced by the two dynamical cores are in very good agreement with the analytical solution. Both models generate similar nonhydrostatic and hydrostatic responses induced by the idealized mountain. The solution of the quasi-analytical linearized problem has been presented in the figure as a qualitative reference. This test case is very sensitive to the choice of the value of the numerical basic-state temperature of the model. The results presented here require $T_{*}=210$ and $240 \mathrm{~K}$ for QE-based dynamical core (GEM-QE) and the operational nonhydrostatic core (GEM-NH), respectively. Historically, this test-case has been a benchmark for identifying any inconsistency in the model formulations as well as the underlying numerics (Husain and Girard 2017; Husain et al. 2019). Therefore, the results for this test-case demonstrated the ability of the QE-formulationbased dynamical core to faithfully represent both the hydrostatic and nonhydrostatic effects for idealized orographic forcing.

\section{d. Global deterministic prediction system (GDPS)}

We have conducted two series of 120-h global forecasts, with $15-\mathrm{km}$ horizontal grid spacing, covering winter and summer 

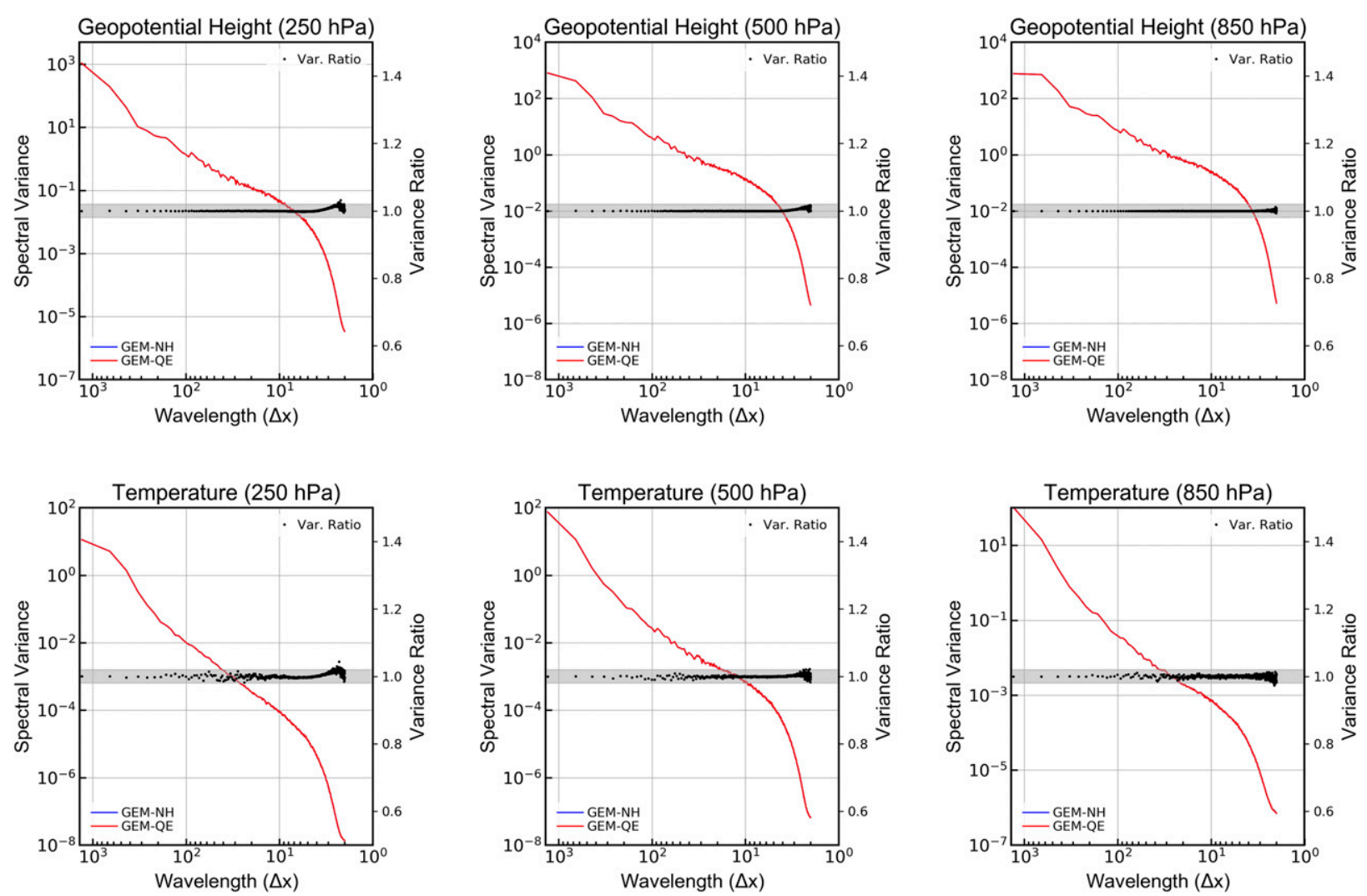

FIG. 9. Spectral variance of (top) GZ and (bottom) TT for 48-h HRDPS forecasts. Results are obtained by averaging the spectra for 10 winter forecasts at three different model levels that in the absence of orography are equivalent to three pressure levels: (left) 250, (center) 500 , and (right) $850 \mathrm{hPa}$. The black circles in the figure denote variance ratios between GEM-QE and GEM-NH for different wavelengths. The shaded area in the figure represents the region bounded by variance ratios of 0.98 and 1.02 , and, consequently, for most length scales the change of variance with GEM-QE is approximately within $2 \%$ of the reference GEM-NH.

periods of the Northern Hemisphere to compare the predictions by the current operational quasi-hydrostatic compressible system of equations (GEM-QH) and the quasi-elastic system of equations (GEM-QE). Both seasonal periods consist of 44 cases and the time of initializations between the successive cases differ by $36 \mathrm{~h}$, starting at 0000 UTC 18 December 2016 and 21 June 2016, respectively, for the winter and summer periods. Other important aspects of the simulation configurations include 84 vertical levels with the model top set at $0.1 \mathrm{hPa}$ and a time-step length of $450 \mathrm{~s}$. Furthermore, after each time step we do a surface pressure adjustment, which consists of a global mass fixer applied to the surface pressure field, which is a prognostic model variable.

We start by comparing the results in the spectral space. Both systems of equations (GEM-QE and GEM-QH) are free of vertically propagating sound waves. It is also important to note that the nonhydrostaticity allowed by the QE equations are not important at $15-\mathrm{km}$ resolution. Therefore, a good agreement in the spectral space between the two systems of equations is expected. Figure 6 shows the spectral variance in the modelpredicted geopotential height and temperature, obtained by averaging over 10 cases, for a forecast lead time of $120 \mathrm{~h}$ during the winter period. The spectral variance from the two systems is virtually indistinguishable. To further complement the comparisons, the ratio of variance between $\mathrm{QE}$ and $\mathrm{QH}$ are also presented in this figure, where the variance ratio for a wavenumber $n$ is computed as

$$
\operatorname{var}_{\text {ratio }}(n)=\operatorname{var}_{\mathrm{QE}}(n) / \operatorname{var}_{\mathrm{QH}}(n) \text {. }
$$

A variance ratio above 1.0 for a given wavenumber implies that GEM-QE leads to increased variance for the associated wavelengths, and vice versa. As can be seen in Fig. 6, for the most part, the change in var ratio remains within $5 \%$, particularly closer to the surface $(850-\mathrm{hPa}$ level). At $100 \mathrm{hPa}$, differences of more than $5 \%$ are present; however, they are restricted to wavenumbers larger than 40 . In other words, for the large scales the two systems produce equivalent levels of variance, whereas the small scales may have relatively larger differences. For the kinetic energy, Fig. 7 demonstrates that the two dynamical cores have the same spectral slope for the entire range of resolved scales. Again, the var ${ }_{\text {ratio }}$ shows no spurious generation or damping in either the kinetic energy or the vertical motion. This indicates that the QE approximation is not expected to lead to any significant changes in the crucial physical processes like convection and convection-driven precipitation. In fact, GEM-QE is found to have equivalent scores for quantitative 

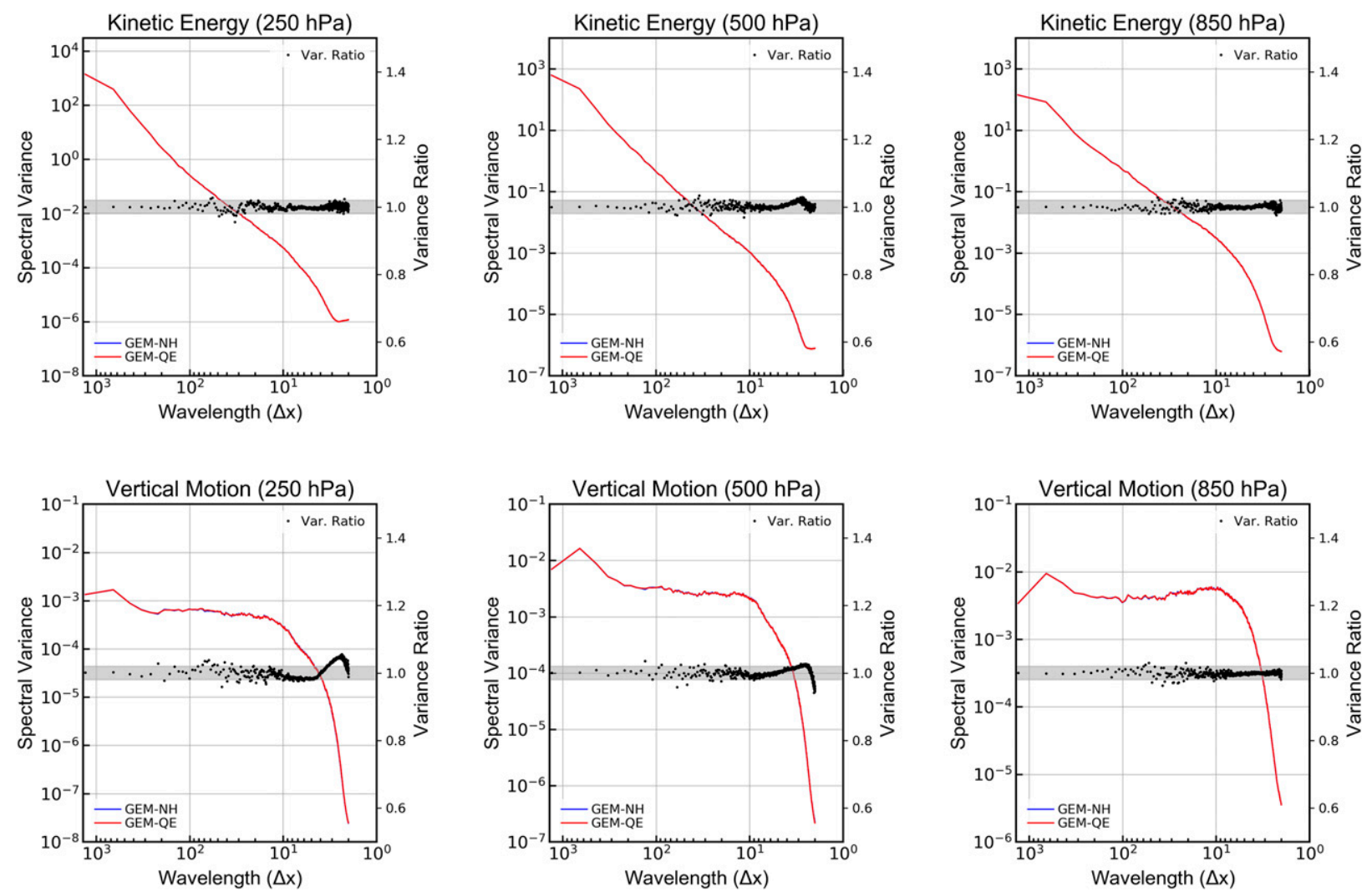

FIG. 10. As in Fig. 9, but for (top) KE and (bottom) WW for 48-h HRDPS forecasts.

precipitation forecast when compared with the operational GEM-QH (not shown).

To assess the impact of the variance at the different modelresolved scales on the quality of the NWP forecasts, we have also compared the model predictions with the ERA5 analyses. The comparisons are done at different pressure levels to compute objective forecast scores. The global average scores associated with a forecast lead time of $120 \mathrm{~h}$ over the winter period for zonal wind (UU), humidity (HU), geopotential height (GZ), and temperature (TT) are presented in Fig. 8. The presence of the red symbols for a pressure level along the left (for bias) and right (for RMSE) edges of the individual panels indicates that the GEM-QE results are relatively better, whereas the blue symbols imply advantage for GEM-QH.
However, at all the pressure levels, the confidence level for the statistical significance of the differences were found to be less than $90 \%$, which implies that the objective scores from the two systems are statistically equivalent. This conclusion is found to hold equally for the summer period (not shown). Overall, the results from the GDPS experiments demonstrate the ability of the new GEM-QE system to produce real world forecasts that are equivalent to the operational GEM-QH system for predominantly hydrostatic scales.

\section{e. High-resolution deterministic prediction system (HRDPS)}

Similar to the GDPS experiments, we have conducted two series of 2-day regional forecasts with $2.5-\mathrm{km}$ horizontal grid
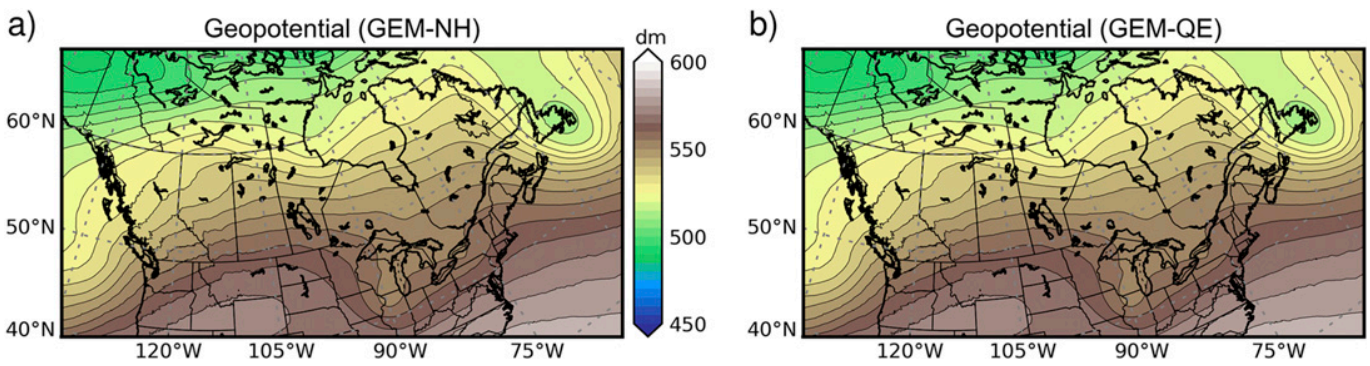

FIG. 11. The geopotential field at $500 \mathrm{hPa}$, for a single case at 48-h lead time with HRDPS valid at 1200 UTC 16 Jan 2017 as predicted by (a) GEM-NH and (b) GEM-QE. 

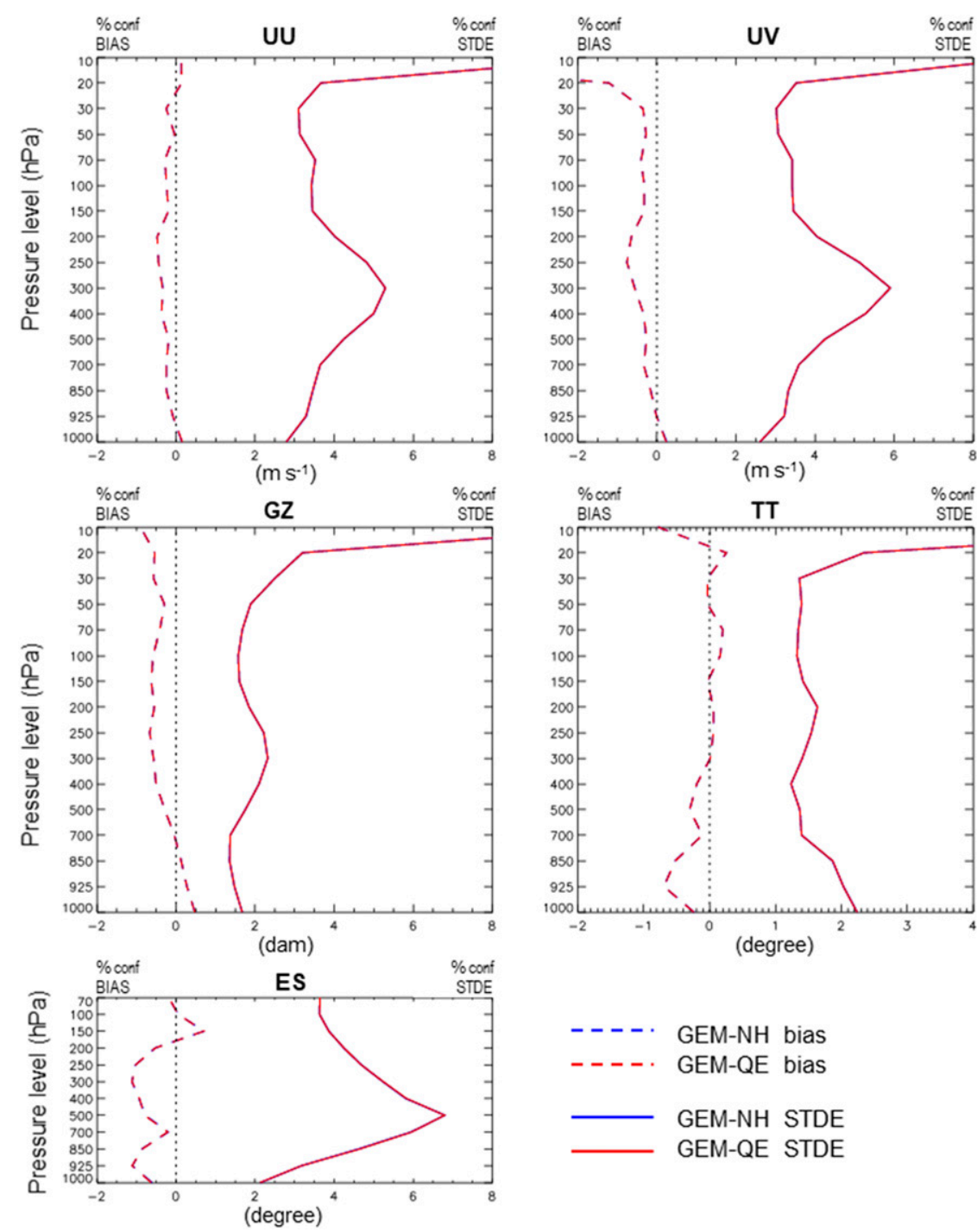

FIG. 12. Objective evaluation of 48-h HRDPS forecasts against radiosonde observations with GEM-NH (blue lines) and with GEM-QE (red lines) for (top left) zonal wind, (top right) wind speed, (center left) geopotential height, (center right) temperature, and (bottom) dewpoint depression. The SDE (solid lines) and bias (dashed lines) are shown. Verification is performed over North America for a set of 44 integrations over the winter period (16 Dec 2016-18 Feb 2017).

spacing, covering winter and summer periods for a limitedarea model (LAM) grid over North America using a timestep length of $60 \mathrm{~s}$, with the new QE-based dynamical core (GEM-QE) and the operational nonhydrostatic core (GEM-NH). The lateral boundary conditions are obtained from ECCCs 10-kmresolution operational RDPS (Regional Deterministic Prediction System). It is important to note that HRDPS also employs vertical lid nesting where the upper boundary conditions are enforced to adhere to the RDPS outputs.

As with the GDPS experiments, comparisons are first made in the spectral space. Figure 9 shows the average spectral variance for the predicted geopotential height and temperature associated with the models based on the two sets of equations for the entire range of scales resolved by the $2.5-\mathrm{km}$ HRDPS. Similarly to GDPS, the two models are found to be spectrally equivalent. Again, the variance ratio between GEM-QE and GEM-NH are presented to complement the information. As the HRDPS simulations are carried over LAM domains with the enforcement of the lateral and upper boundary conditions, this leads to a tighter control over the model predictions relative to the global simulations. Consequently, the variance ratio show smaller deviations from 1.0, and for most of the model-resolved scales, its divergence is found to be within $2 \%$. The spectra and variance ratio of kinetic energy and vertical velocity are displayed in Fig. 10. In addition to having minimal deviations in 

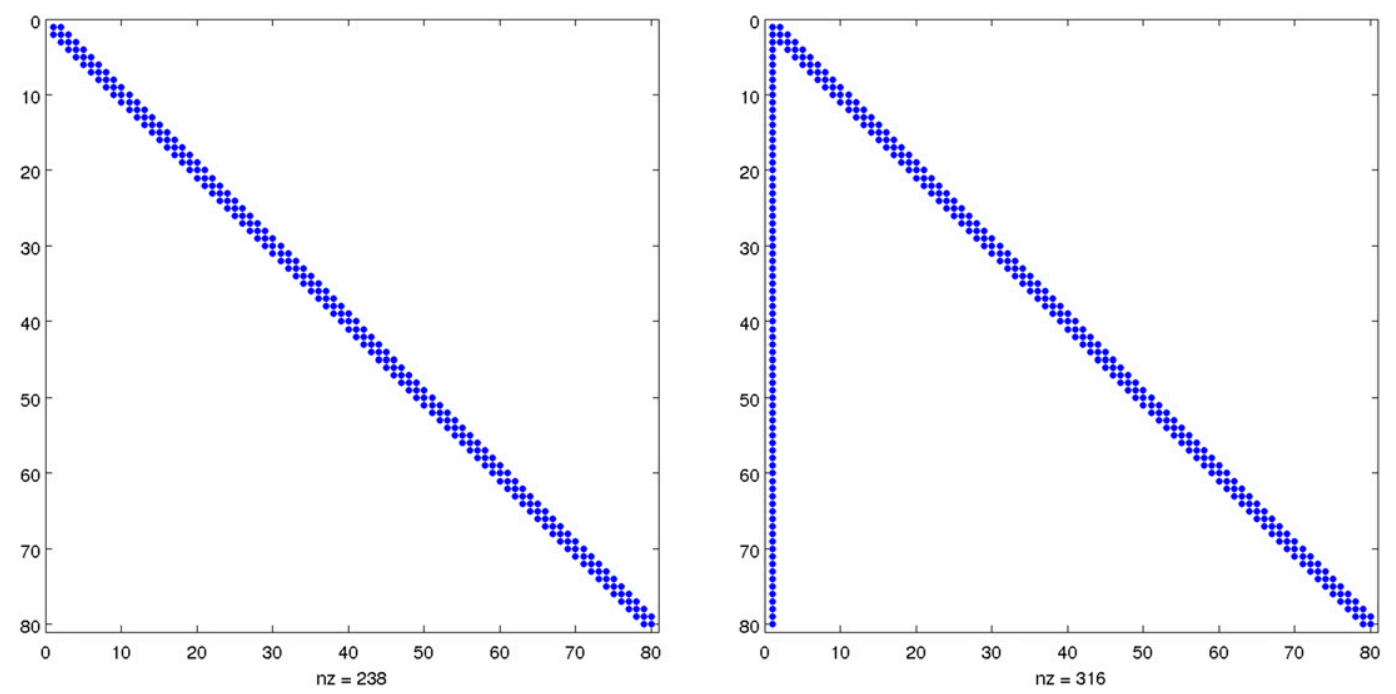

FIG. 13. The sparsity of the vertical structure matrix of (left) GEM-NH and (right) GEM-QE.

the variance ratio, the two dynamical cores are found to have indistinguishable spectral slopes of kinetic energy. The spectra of vertical motion from the two dynamical cores also reveal no discernible difference. Although GEM-NH permits acoustic modes, the use of implicit discretization slows down the lowenergy sound waves and the use of off-centering along with second-order horizontal diffusion applied to the wind along the coordinate surfaces largely eliminate the acoustic signature. This explains the spectral similarity between GEM-QE and GEM-NH for HRDPS.

For both GEM-NH and GEM-QE, Fig. 11 shows the geopotential field at $500 \mathrm{hPa}$ for a single case with a forecast lead time of $48 \mathrm{~h}$ valid at 1200 UTC 16 January 2017. The figure reveals that although there are small differences in the spectral space leading to divergences in the variance ratio from 1 , in the physical space that do not result in any significant difference between the two models. Even though we can discern small differences between the two models, when it is considered in conjunction with the spectral variance results (Figs. 9 and 10), it is safe to state that GEM-QE does not lead to any spurious noise generation or damping in comparison with the operational GEM-NH.

Objective forecast scores were computed by comparing the model predictions with radiosonde observations. Figure 12 represents the domain-average scores of 48-h forecasts over the winter period from 44 cases for zonal wind (UU), wind speed (UV), geopotential height (GZ), temperature (TT), and dewpoint depression (ES). These scores show that there is no statistically significant difference either in the standard deviation of error (SDE) or the bias between the two dynamical cores. To summarize, the HRDPS experiments demonstrate that, for strongly nonhydrostatic scenarios, the GEM-QE system is capable of producing forecasts that are equivalent to the existing GEM-NH system in terms of accuracy.

\section{f. Computational cost}

The differences in the equations between the QE and the operational dynamical cores, when subjected to the discretizations and elimination of variables, leads to slightly different elliptic problems that can result in a small difference in the computational performance of the respective dynamical cores (see appendix B). It is important to note that the largest computational overhead associated with any GEM dynamical core is attributable to the elliptic solver. As shown in appendix B, there are differences in the elliptic operator (matrix) and the rhs of the elliptic equation [Eq. (B19)] due to modifications in the TFC between the two dynamical cores. Figure 13 shows that the vertical structure matrices of the two models are slightly different. The term $\left(\delta_{\zeta} M-M_{a} \delta_{\zeta}\right)$ appearing in the elliptic equation [Eq. (B19)] of the GEM-QE model is nonzero only for the first vertical level $k=1$. This explains the presence of a nonzero additional column that makes the vertical structure matrix nontridiagonal unlike the operational GEM model (both GEM-NH and GEM-QH) (see Fig. 13). Eigenmodes of the vertical structure matrix are precomputed by using the same "dsyg" subroutine of the Linear Algebra Package (LAPACK). Consequently, no significant difference in the computational cost is expected with respect to the elliptic solvers between the QE and the operational models. Another important difference between the two dynamical cores is the presence of the operator $\left(\delta_{\zeta}+\kappa M_{a}\right)^{-1}$ in GEM-QE [see Eq. (B19)]. This numerical inversion adds a small computational overhead during the algebraic reduction of equations (see appendix B) in order to compute the rhs term of the elliptic equation.

Table 1 shows the wall-clock time for 144-h GDPS simulations using 800 CPUs of the Cray XC40 supercomputer and 48-h HRDPS simulations using 1750 CPUs. In all of these simulations, the direct solver was used to solve the elliptic problem. Because of its small computational cost relative to its iterative counterpart, the direct solver is always used with the GEM model for all the current operational configurations at ECCC. Results presented in the table show the mean dynamics runtime for 88 runs that differ in their times of initialization, for both GDPS and HRDPS experiments (44 winter cases and 44 
TABLE 1. Average running time (s) of GEM-QE, GEM-QH, and GEM-NH dynamical cores for 144-h GDPS $(\Delta t=450 \mathrm{~s})$ and 48 -h $\operatorname{HRDPS}(\Delta t=60 \mathrm{~s})$ simulations.

\begin{tabular}{lc}
\hline \hline \multicolumn{1}{c}{ Model } & Dynamics runtime \\
\hline GEM-QE HRDPS & $3460 \mathrm{~s}$ \\
GEM-NH HRDPS & $3390 \mathrm{~s}$ \\
GEM-QE GDPS & $1700 \mathrm{~s}$ \\
GEM-H GDPS & $1685 \mathrm{~s}$ \\
\hline
\end{tabular}

summer cases). Table 1 shows that the dynamics of GEM-QE leads to a slight increase in computational time for these NWP tests (less than 1\% for GDPS and 2\% for HRDPS). When the complete computational cost for these experiments- that include the cost of physical parameterizations - are considered, the difference is even more negligible. Therefore, we can conclude with confidence that the new core does not incur any significant additional costs.

\section{Summary}

An optional dynamical core based on the QE system of equations has been developed within ECCC's GEM atmospheric model. The QE approach requires decoupling of the feedback between the velocity divergence and the nonhydrostatic pressure deviations in the continuity equation. Through linear analysis, it has been demonstrated that the QE dynamical system - as implemented in GEM-does not contain any vertically propagating acoustic modes in its solution. As it primarily requires modification in the existing continuity equation and two diagnostic relations, the implementation of the QE dynamical core led to relatively minor changes in the existing informatics code of the operational GEM model. More important, the implementation of the QE approximation in GEM does not lead to any significant increase in the computational cost. In Voitus et al. (2019), for example, the major changes done to their existing model, in order to implement the QE approximation, led to a considerable increase in the computational overhead and also increased the complexity of their QE dynamical core formulation.

A number of tests-covering both two-dimensional idealized test cases and three-dimensional NWP systems-have been carried out to evaluate the performance of the novel QE dynamical core. The theoretical tests demonstrated that the QE dynamical core performs equally well in representing the buoyancy effects and orography-induced gravity waves. For the NWP tests, the predictions of the QE dynamical core are compared in the spectral space with those obtained from the operational model for both global (hydrostatic) and regional high-resolution (nonhydrostatic) forecasts. The results show excellent agreement over the entire range of scales resolved by the models. Additionally, the predictions from the new QE core are evaluated against the operational forecasts through objective scores computed by comparing the model outputs with ERA5 analyses and radiosonde observations. For both global (hydrostatic) and regional (nonhydrostatic) predictions, the new dynamical core is found to result in statistically equivalent scores, when compared with the operational model.
Based on these results, the QE system of equations can be adopted in future for operational NWP as a soundproof system of equations to produce reliable forecasts at all meteorological scales of interest with negligible changes for the computational overhead. In addition, the possibility of maintaining a single informatics code for the model, instead of multiple models, i.e., GEM-QH and GEM-NH, certainly represents another important advantage of GEM-QE. Finally, the QE system of equations filters a priori unwanted modes of motion associated with the fully compressible system, while correctly capturing all other dynamical modes of interest. As has been stated by Benacchio et al. (2014), in the context of a soundproof-tocompressible system, the QE system could therefore be beneficial for model initializations and data assimilation because of its ability to filter out the undesirable and unbalanced modes from NWP forecasts.

Acknowledgments. The authors thank their colleagues at RPN-A for their valuable suggestions and comments during the development of the GEM-QE.

Data availability statement. The dataset generated for this study is too large to share publicly. However, the data will be retained by the corresponding author and, should there be any request, reasonable efforts will be made to share the data.

\section{APPENDIX A}

\section{Derivation of the QE Equations in the Modified Mass-Based TFC}

Here we will derive only the equations affected by the use of the new TFC, that is, the continuity equation and the two diagnostic equations (the $\mu$ equation and the hydrostatic equation).

The continuity equation is written as

$$
\frac{d}{d t} \ln \left(\rho \frac{\partial z}{\partial \zeta}\right)+\nabla_{\zeta} \cdot \mathbf{V}_{h}+\frac{\partial \dot{\zeta}}{\partial \zeta}=0
$$

By using the relations $\partial \tilde{p} / \partial z=-g \tilde{\rho}$ and $\rho=\tilde{\rho} \exp [(1-\kappa) q]$, the first term could be transformed as

$$
\frac{d \ln \left(\frac{\rho}{\tilde{\rho}} \tilde{\rho} \frac{\partial z}{\partial \zeta}\right)}{d t}=\frac{d \ln \left[e^{(1-\kappa) q} \frac{\partial \tilde{p}}{\partial \zeta}\right]}{d t} .
$$

Later, by using $\ln \tilde{p}=\zeta+B s$, the continuity equation takes the following form:

$$
\frac{d}{d t}\left[B s+\ln \left(1+\frac{\partial B}{\partial \zeta} s\right)\right]+\nabla_{\zeta} \cdot \mathbf{V}_{h}+\frac{\partial \dot{\zeta}}{\partial \zeta}+\dot{\zeta}+(1-\kappa) \frac{d q}{d t}=0
$$

By neglecting the last term $(1-\kappa) d q / d t$ involving the nonhydrostatic pressure tendency, the mass continuity equation in the $\mathrm{QE}$ system of equations is approximated by

$$
\frac{d}{d t}\left[B s+\ln \left(1+\frac{\partial B}{\partial \zeta} s\right)\right]+\nabla_{\zeta} \cdot \mathbf{V}_{h}+\frac{\partial \dot{\zeta}}{\partial \zeta}+\dot{\zeta}=0
$$



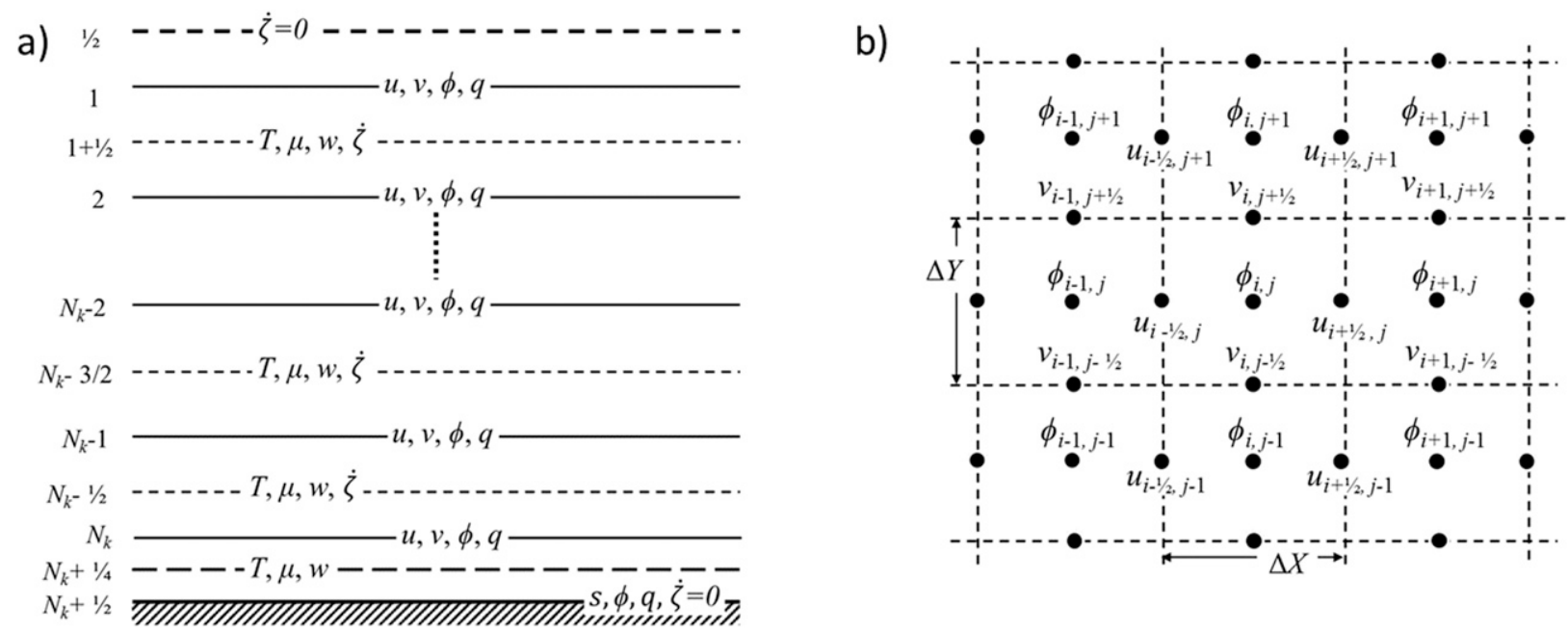

FIG. B1. The (a) vertical Charney-Phillips grid and (b) horizontal Arakawa C grid.

Before deriving the equation for $\mu$, it is important to note that the variable $\mu$ is defined as the ratio of the vertical acceleration to the gravitational acceleration; that is, $\mu=(d w / d t) / g$. The equation of vertical velocity is written as

$$
\frac{d w}{d t}+R T\left(\frac{\partial z}{\partial \zeta}\right)^{-1} \frac{\partial \ln p}{\partial \zeta}+g=0
$$

The equation for $\mu$ is then obtained by plugging in the definition of $\mu$ in the equation of vertical velocity, which gives

$$
1+\mu-e^{\kappa q}\left[1+\frac{\frac{\partial q}{\partial \zeta}}{1+\frac{\partial B}{\partial \zeta} s}\right]=0
$$

where we have used $\partial \ln p / \partial \ln \tilde{p}=1+(\partial q / \partial \ln \tilde{p}),(\partial \ln \tilde{p} / \partial z)=$ $-g /(R \tilde{T}), T=\tilde{T} e^{\kappa q}$, and $\ln \tilde{p}=\zeta+B s$.

The hydrostatic equation $\partial \tilde{p} / \partial z=-g \tilde{\rho}$, the last equation to be derived, is then transformed as

$$
\frac{T}{T_{*}}-e^{\kappa q}\left\{1-\frac{\partial_{\zeta}\left[\phi^{\prime}+R T_{*}(B s)\right]}{R T_{*}\left[1+\partial_{\zeta}(B s)\right]}\right\}=0,
$$

where we have used $T=\tilde{T} e^{\kappa q}, \phi=\phi_{*}+\phi^{\prime}, \partial \phi_{*} / \partial \zeta=-R T_{*}$, and $\ln (\tilde{p})=\zeta+B s$.

\section{APPENDIX B}

\section{Numerical Solution of the QE System of Equations}

The description of the numerical solution method for the QE system of equations presented in this section largely follows that of Husain et al. (2019), and is similar to the one used in the current operational GEM model (Qaddouri and Lee 2011; Girard et al. 2014). However, there are differences in some equation forms and the way of reducing the algebraic systems obtained, by temporal and spatial discretizations, to a single three-dimensional elliptic boundary-value problem with respect to a single variable. The relevant details are presented in this appendix.

\section{a. Spatial discretization}

The horizontal and vertical grids used in the GEM model are displayed in Fig. B1. In the horizontal plane, a staggered Arakawa C grid (Arakawa 1988) is used, and the vertical discretization is based on a staggered Charney-Phillips grid (Charney and Phillips 1953). The centered finite-difference method is used for the space discretization in both the horizontal and vertical directions. Furthermore, in the operational GEM model, the regional grid systems are based on latitudelongitude limited-area grids whereas the global system is based on a yin-yang grid approach (Qaddouri and Lee 2011).

\section{b. Temporal discretization}

The time discretization used to integrate the frictionless adiabatic QE equations is based on a two time-level iterativeimplicit semi-Lagrangian approach (Côté and Staniforth 1988; Husain and Girard 2017). In general, the prognostic equations in the QE system of equations can be expressed as

$$
\frac{d F_{i}}{d t}-G_{i}=0
$$

where $F_{i}$ represents the prognostic quantity for an equation $i$ within the system and $G_{i}$ includes the remaining terms, some of which are nonlinear. Such an equation is approximated by time differences and weighted averages along a trajectory determined by an approximate solution to the kinematic equations:

$$
\begin{aligned}
\frac{d \mathbf{r}_{h}}{d t} & =\mathbf{V}_{h} \quad \text { and } \\
\frac{d \zeta}{d t} & =\dot{\zeta},
\end{aligned}
$$

where $\left(\mathbf{r}_{h}, \zeta\right)$ and $\left(\mathbf{V}_{h}, \dot{\zeta}\right)$ are the three-dimensional position and velocity, respectively. Through the application of implicit two-time-level discretization along the computed trajectory, 
Eq. (B1) may be integrated following the motion over a time interval $\Delta t$ leading to

$$
\frac{F_{i}^{A}-F_{i}^{D}}{\Delta t}-\frac{1+b_{i}}{2} G_{i}^{A}-\frac{1-b_{i}}{2} G_{i}^{D}=0,
$$

where the superscripts $A$ and $D$ represent the arrival and departure positions, respectively, and the parameter $b_{i}$ represents the off-centering weight factor. For the GEM model operational configurations, $b_{i}$ is set to 0.2 . Furthermore, cubic interpolation is used for the upstream computations of both the wind field and the dynamical source terms $G_{i}^{D}$ for numerical consistency (Husain and Girard 2017).

\section{c. The elliptic problem by a reduction method}

To solve the spatially discretized QE system of equations, we rearrange the unknown terms in each nonlinear equation of the form in Eq. (B3) into separated linear and nonlinear parts as

$$
L_{i}=R_{i}-N_{i},
$$

where $L_{i}$ contains the linearized left-hand side (lhs) terms, $N_{i}$ are the remaining nonlinear terms along with the Coriolis and metric terms, and $R_{i}$ are the known quantities. The linear parts of the rearranged QE equations from Eq. (B4) are as follows:

$$
\begin{aligned}
\mathbf{L}_{\mathbf{v}_{h}} & =\frac{\mathbf{V}_{h}}{\tau}+\nabla_{\zeta} P, \\
L_{w} & =\frac{w}{\tau}-g \mu, \\
L_{C} & =\nabla_{\zeta} \cdot \mathbf{V}_{h}+\delta_{\zeta} Y+\bar{Y}^{\zeta}, \\
L_{T} & =\frac{T^{\prime}}{\tau T_{*}}-\kappa\left(Y+\frac{\bar{q}^{\zeta}}{\tau}\right), \\
L_{\phi} & =\frac{\bar{P}^{\zeta}}{\tau}-R T_{*}\left(Y+\bar{q}^{\zeta}\right)-g w, \\
L_{H} & =\frac{T^{\prime}}{T_{*}}+\frac{\delta_{\zeta} P}{R T_{*}}-\left(\delta_{\zeta} q+\kappa \bar{q}^{\zeta}\right), \quad \text { and } \\
L_{\mu} & =\mu-\left(\delta_{\zeta} q+\kappa \bar{q}^{\zeta}\right),
\end{aligned}
$$

where the vertical derivative is replaced by simple finite differences represented here by the symbol $\delta_{\zeta}$, and the spatial averaging operation along $\zeta$ is represented by the overline operator $\overline{()^{\zeta}}$. Note that $\nabla_{\zeta}$ is now the spatially discretized horizontal gradient operator along $\zeta$. As in Côté and Staniforth (1988), the solution of the system of equations of the type (B4) requires two nonlinear iterations, the minimum for stability and the maximum for practical operational use. Since there are two Crank-Nicolson iterations, where the $R_{i}$ terms are reevaluated during each iteration at the departure positions, the system of equations of the type in Eq. (B4) is solved four times during each dynamical time step.

The solution of the system of equations of the type in Eq. (B4) with the lhs given by Eqs. (B5)-(B11) can be algebraically reduced to the solution of a one three-dimensional elliptic boundary-value problem for the variable $P$. The other model variables are obtained through back substitution. We first eliminate $\mathbf{V}_{h}, \mu$, and $T$, leaving four equations with four unknowns, $w, q, P$, and $Y$ :

$$
\begin{aligned}
& L_{c}^{\prime}=\nabla_{\zeta} \cdot \mathbf{L}_{\mathbf{v}_{h}}-\frac{L_{c}}{\tau}=\nabla_{\zeta}^{2} P-\frac{1}{\tau}\left(\delta_{\zeta} Y+\bar{Y}^{\zeta}\right), \\
& L_{w}^{\prime}=L_{w}+g L_{\mu}=\frac{w}{\tau}-g\left(\delta_{\zeta} q+\kappa \bar{q}^{\zeta}\right), \\
& L_{T}^{\prime}=L_{T}-\frac{L_{H}}{\tau}=-\frac{\delta_{\zeta} P}{\tau R T_{*}}+\frac{\delta_{\zeta} q}{\tau}-\kappa Y, \quad \text { and } \\
& L_{\phi}=\frac{\bar{P}^{\zeta}}{\tau}-R T_{*}\left(Y+\bar{q}^{\zeta}\right)-g w .
\end{aligned}
$$

At this stage, we can eliminate $w$ followed by $q$, leaving $Y$ and $P$ as

$$
\begin{aligned}
L_{T}^{\prime \prime}= & \frac{1}{\tau(\kappa+\varepsilon)}\left(L_{T}^{\prime}+\frac{L_{w}^{\prime}}{g \tau}-\frac{L_{\phi}}{g^{2} \tau^{2}}\right) \\
= & -\Gamma\left(\delta_{\zeta} P+\varepsilon \bar{P}^{\zeta}\right)-\frac{1}{\tau}\left(Y+\frac{\bar{q}^{\zeta}}{\tau}\right) \quad \text { and } \\
L_{\mathrm{CB}}= & \delta_{\zeta} L_{T}^{\prime \prime}+\frac{1}{\tau}{\overline{L_{T}^{\prime}}}^{\zeta}=-\Gamma\left[\delta_{\zeta} \delta_{\zeta} P+\kappa \overline{\delta_{\zeta} P^{\zeta}}+\varepsilon\left(\overline{\delta_{\zeta} P^{\zeta}}-\delta_{\zeta} \bar{P}^{\zeta}\right)\right] \\
& -\frac{1}{\tau}\left(\delta_{\zeta} Y+\kappa \bar{Y}^{\zeta}\right),
\end{aligned}
$$

where $\varepsilon=R T_{*} /\left(g^{2} \tau^{2}\right)$ and $\Gamma=1 /\left[\tau^{2} R T_{*}(\kappa+\varepsilon)\right]$. Last, we eliminate $Y$ and obtain an elliptic boundary value problem for the variable $P$, with its lhs given by

$$
\begin{aligned}
L_{p}= & L_{c}^{\prime}-\left(\delta_{\zeta}+M_{a}\right)\left(\delta_{\zeta}+\kappa M_{a}\right)^{-1} L_{\mathrm{CB}} \\
L_{p}= & \nabla_{\zeta}^{2} P+\Gamma\left(\delta_{\zeta}+M_{a}\right) \delta_{\zeta} P-\Gamma \varepsilon\left(\delta_{\zeta}+M_{a}\right)\left(\delta_{\zeta}+\kappa M_{a}\right)^{-1} \\
& \times\left(\delta_{\zeta} M-M_{a} \delta_{\zeta}\right) P,
\end{aligned}
$$

where $M_{a}$ and $M$ are the spatial averaging operators in the $\zeta$ coordinate on the thermodynamic and the momentum levels, respectively. It is important to note that the same algebraic reductions are also applied to nonlinear right-hand sides $R_{i}-N_{i}$ of the equations.

\section{d. Initial and boundary conditions}

The numerical solution of the QE dynamical core needs initial values of all the prognostic variables. We use ECCC's current operational data assimilation system, which provides analyzed initial values for the horizontal wind components $u$ and $v$, temperature $T$, and surface pressure $p_{s}$. At present, the remaining prognostic variables $w, \dot{\zeta}$, and $q$ are initialized in a diagnostic way. For the boundary conditions at the upper and lower boundaries we use $\dot{\zeta}_{T}=\dot{\zeta}_{S}=0$. Furthermore, for limited-area grids, the lateral boundary conditions are obtained from the driving fields.

\section{e. Solution of the elliptic problem}

The three-dimensional elliptic boundary-value problem to be solved four times at each time step can be written as 


$$
\begin{aligned}
& \nabla_{\zeta}^{2} P+\Gamma\left(\delta_{\zeta}+M_{a}\right) \delta_{\zeta} P-\Gamma \varepsilon\left(\delta_{\zeta}+M_{a}\right)\left(\delta_{\zeta}+\kappa M_{a}\right)^{-1} \\
& \quad \times\left(\delta_{\zeta} M-M_{a} \delta_{\zeta}\right) P=\mathbf{R}_{E}
\end{aligned}
$$

where $P$ is the unknown and $\mathbf{R}_{E}$ contains the nonlinear terms along with the explicit rhs terms. Two numerical solver approaches are available for solving the elliptic problem - direct and iterative. Although in this paper we will only present the direct solver, it is important to note that the iterative solver is based on the preconditioned flexible generalized minimal residual (PFGMRES) method (Saad 1993; Qaddouri and Lee 2010). The direct solver begins by vertical separation where the rhs $\mathbf{R}_{E}$ is expanded in terms of the eigenvectors of the operator $\Gamma\left(\delta_{\zeta}+M_{a}\right) \delta_{\zeta}-\Gamma \varepsilon\left(\delta_{\zeta}+M_{a}\right)\left(\delta_{\zeta}+\kappa M_{a}\right)^{-1}\left(\delta_{\zeta} M-M_{a} \delta_{\zeta}\right) . \mathrm{We}$ will refer to this operator as the vertical structure operator. The sparsity of the matrix representing this operator is shown in Fig. 13. The matrix representing the vertical structure operator in the current GEM model is simply tridiagonal, whereas the QE approximation adds an additional first column that reflects a vertical coupling between the topmost level and the other vertical levels. The vertical separation reduces Eq. B19 to a set of $N_{K}$ independent positive definite horizontal Helmholtz problems of the form

$$
\left(\mathbf{c}-\nabla_{\zeta}^{2}\right) \tilde{P}=\tilde{\mathbf{R}}_{E},
$$

where $N_{k}$ is the total number of vertical levels, $\tilde{P}$ and $\tilde{\mathbf{R}}_{E}$ are the vertical projections of $P$ and $\mathbf{R}_{E}$, respectively, and $\mathbf{c}$ is a diagonal matrix in which the entries are the eigenvalues of the vertical structure operator. The solver then continues with the horizontal solution of Eq. (B20) by expanding $\tilde{\mathbf{R}}_{E}$ in terms of the eigenvectors of the longitude component of the twodimensional operator $\nabla_{\zeta}^{2}$. This leads to $N_{i}$ independent tridiagonal problems of dimension $N_{j}$, where $N_{i}$ and $N_{j}$ are the number of grid points along longitude and latitude direction, respectively. The tridiagonal problems are solved by using Gaussian elimination method without pivoting, and finally the three-dimensional solution $P$ is obtained by Fourier synthesis (Qaddouri et al. 1999). The direct solver uses Fast Fourier Transform to compute the horizontal solution and this makes this direct solver computationally more efficient than most iterative methods, particularly for the spatial resolutions of the current operational NWP systems at ECCC.

\section{REFERENCES}

Arakawa, A., 1988: Finite difference methods in climate modeling. Physically-Based Modeling and Simulation of Climate and Climate Change, M. E. Schlesinger, Ed., Springer, 79-168.

- , and C. S. Konor, 2009: Unification of the anelastic and quasihydrostatic system of equations. Mon. Wea. Rev., 137, 710726, https://doi.org/10.1175/2008MWR2520.1.

Batchelor, G. K., 1953: The condition for dynamical similarity of motions of a frictionless perfect-gas atmosphere. Quart. J. Roy. Meteor. Soc., 79, 224-235, https://doi.org/10.1002/ qj.49707934004.

Benacchio, T., and R. Klein, 2016: A doubly blended model for multiscale atmospheric dynamics. J. Atmos. Sci., 73, 11791186, https://doi.org/10.1175/JAS-D-15-0323.1.

— W. P. O'Neill, and R. Klein, 2014: A blended soundproofto-compressible numerical model for small- to mesoscale atmospheric dynamics. Mon. Wea. Rev., 142, 4416-4438, https://doi.org/10.1175/MWR-D-13-00384.1.

Charney, J., and N. A. Phillips, 1953: Numerical integration of the quasi-geostrophic equations for barotropic and simple baroclinic flows. J. Meteor., 10, 71-99, https://doi.org/10.1175/15200469(1953)010<0071:NIOTQG > 2.0.CO;2.

Côté, J., and A. Staniforth, 1988: A two-time-level semi-Lagrangian semi-implicit scheme for spectral models. Mon. Wea. Rev., 116, 2003-2012, https://doi.org/10.1175/1520-0493(1988)116<2003: ATTLSL $>2.0 . \mathrm{CO} ; 2$.

Davies, T., A. Staniforth, N. Wood, and J. Thuburn, 2003: Validity of anelastic and other equation sets as inferred from normalmode analysis. Quart. J. Roy. Meteor. Soc., 129, 2761-2775, https://doi.org/10.1256/qj.02.1951.

Durran, D. R., 1989: Improving the anelastic approximation. J. Atmos. Sci., 46, 1453-1461, https://doi.org/10.1175/15200469(1989)046<1453:ITAA > 2.0.CO;2.

_ 2008: A physically motivated approach for filtering acoustic waves from the equations governing compressible stratified flow. J. Fluid Mech., 601, 365-379, https://doi.org/10.1017/ S0022112008000608.

Giraldo, F. X., and M. Restelli, 2008: A study of spectral element and discontinuous Galerkin methods for the Navier-Stokes equations in nonhydrostatic mesoscale atmospheric modeling: Equation sets and test cases. J. Comput. Phys., 227, 3849-3877, https://doi.org/10.1016/j.jcp.2007.12.009.

Girard, C., and Coauthors, 2014: Staggered vertical discretization of the Canadian Environmental Multiscale (GEM) model using a coordinate of the log-hydrostatic-pressure type. Mon. Wea. Rev., 142, 1183-1196, https://doi.org/10.1175/MWR-D13-00255.1.

Husain, S. Z., and C. Girard, 2017: Impact of consistent semiLagrangian trajectory calculations on numerical weather prediction performance. Mon. Wea. Rev., 145, 4127-4150, https://doi.org/10.1175/MWR-D-17-0138.1.

$\longrightarrow,-$ A. Qaddouri, and A. Plante, 2019: A new dynamical core of the Global Environmental Multiscale (GEM) model with a height-based terrain-following vertical coordinate. Mon. Wea. Rev., 147, 2555-2578, https://doi.org/10.1175/ MWR-D-18-0438.1.

,-- L. Separovic, A. Plante, and S. Corvec, 2020: On the progressive attenuation of fine-scale orography contributions to the vertical coordinate surfaces within a terrain-following coordinate system. Mon. Wea. Rev., 148, 4143-4158, https:// doi.org/10.1175/MWR-D-20-0085.1.

Konor, C. S., 2014: Design of a dynamical core based on the nonhydrostatic "unified system" of equations. Mon. Wea. Rev., 142, 364-385, https://doi.org/10.1175/MWR-D-13-00187.1.

Laprise, R., 1992: The Euler equations of motion with hydrostatic pressure as an independent variable. Mon. Wea. Rev., 120, 197-207, https://doi.org/10.1175/1520-0493(1992)120<0197: TEEOMW $>2.0 . \mathrm{CO} ; 2$.

Nance, L., and D. Durran, 1994: A comparison of the accuracy of three anelastic systems and the pseudo-incompressible system. J. Atmos. Sci., 51, 3549-3565, https://doi.org/10.1175/15200469(1994)051<3549:ACOTAO > 2.0.CO;2.

Ogura, Y., and N. A. Phillips, 1962: Scale analysis of deep and shallow convection in the atmosphere. J. Atmos. Sci., 19, 173-179, https://doi.org/10.1175/1520-0469(1962)019<0173: SAODAS $>2.0 . \mathrm{CO} ; 2$.

Qaddouri, A., and V. Lee, 2010: The elliptic solvers in the Canadian limited area forecasting model GEM-LAM. Modeling Simulation and Optimization-Tolerance and 
Operational Control, S. Cakaj, Ed., InTechOpen, 1-17, https://doi.org/10.5772/9038.

_, and — 2011: The Canadian global environmental multiscale model on the Yin-Yang grid system. Quart. J. Roy. Meteor. Soc., 137, 1913-1926, https://doi.org/10.1002/qj.873.

— J. Jôté, and M. Valin, 1999: A parallel direct 3D elliptic solver. High Performance Computing Systems and Applications, A. Pollard, D. Mewhort, and D. Weaver, Eds., Kluwer Academic, 429-442.

Randall, D. A., 2000: The anelastic and Boussinesq approximations. Colorado State University Selected Notes of David A. Randall, 85-98, https://clouds.eos.ubc.ca/ phil/courses/AneBous.pdf.

Robert, A., 1993: Bubble convection experiments with a semiimplicit formulation of the Euler equations. J. Atmos. Sci., 50, 1865-1873, https://doi.org/10.1175/1520-0469(1993)050<1865: BCEWAS $>2.0 . C O ; 2$.

Saad, Y., 1993: A flexible inner-outer preconditioned GMRES algorithm. SIAM J. Sci. Comput., 14, 461-469, https://doi.org/ $10.1137 / 0914028$.

Schär, C., D. Leuenberger, O. Fuhrer, D. Lüthi, and C. Girard, 2002: A new terrain-following vertical coordinate formulation for atmospheric prediction models. Mon. Wea. Rev., 130, 2459-2480, https://doi.org/10.1175/1520-0493(2002)130<2459: ANTFVC $>2.0 . C O ; 2$.

Smolarkiewicz, P., L. Margolin, and A. Wyszogrodzki, 2001: A class of nonhydrostatic global models. J. Atmos. Sci., 58, 349-364, https://doi.org/10.1175/1520-0469(2001)058<0349: ACONGM $>2.0 . \mathrm{CO} ; 2$.

Straka, J. M., R. Wilhelmson, L. Wicker, J. Anderson, and K. Droegemeier, 1993: Numerical solutions of a non-linear density current: A benchmark solution and comparisons. Int. J. Numer. Methods Fluids, 17, 1-22, https://doi.org/10.1002/ fld.1650170103.

Ullrich, P. A., and Coauthors, 2017: DCMIP2016: A review of nonhydrostatic dynamical core design and intercomparison of participating models. Geosci. Model Dev., 10, 4477-4509, https://doi.org/10.5194/gmd-10-4477-2017.

Voitus, F., P. Bénard, C. Kühnlein, and N. P. Wedi, 2019: Semiimplicit integration of the unified equations in a mass-based coordinate: Model formulation and numerical testing. Quart. J. Roy. Meteor. Soc., 145, 3387-3408, https://doi.org/ 10.1002/qj.3626. 